\title{
A very young star forming region detected by the ISOPHOT Serendipity Survey ${ }^{\star}$
}

\author{
O. Krause, D. Lemke, L. V. Tóth, U. Klaas, M. Haas, M. Stickel, and R. Vavrek \\ Max-Planck-Institut für Astronomie (MPIA), Königstuhl 17, 69117 Heidelberg, Germany \\ Received 26 August 2002 / Accepted 12 September 2002

\begin{abstract}
We present a multi-wavelength study of the star forming region ISOSS J 20298+3559, which was identified by a cross-correlation of cold compact sources from the $170 \mu \mathrm{m}$ ISOPHOT Serendipity Survey (ISOSS) database coinciding with objects detected by the MSX, 2MASS and IRAS infrared surveys. ISOSS J 20298+3559 is associated with a massive dark cloud complex $\left(M \sim 760 M_{\odot}\right)$ and located in the Cygnus X giant molecular cloud. We derive a distance of $1800 \mathrm{pc}$ on the basis of optical extinction data. The low average dust temperature $(T \sim 16 \mathrm{~K})$ and large mass $\left(M \sim 120 M_{\odot}\right)$ of the dense inner part of the cloud, which has not been dispersed, indicates a recent begin of star formation. The youth of the region is supported by the early evolutionary stage of several pre- and protostellar objects discovered across the region: I) two candidate Class 0 objects with masses of 8 and $3.5 M_{\odot}$, II) a gravitationally bound, cold $(T \sim 12 \mathrm{~K})$ and dense $\left(n\left(\mathrm{H}_{2}\right) \sim 2 \times 10^{5} \mathrm{~cm}^{-3}\right)$ cloud core with a mass of $50 M_{\odot}$ and III) a Herbig B2 star with a mass of $6.5 M_{\odot}$ and a bolometric luminosity of $2200 L_{\odot}$, showing evidence for ongoing accretion and a stellar age of less than 40000 years. The dereddened SED of the Herbig star is well reproduced by an accretion disc + star model. The externally-heated cold cloud core is a good candidate for a massive pre-protostellar object. The star formation efficiency in the central cloud region is about $14 \%$.
\end{abstract}

Key words. stars: formation - ISM: dust, extinction - ISM: clouds - ISM: individual objects: ISOSS J 20298+3559

\section{Introduction}

It is a challenge to identify massive young stellar objects during their early evolution. The youngest protostars form deeply embedded in their cold $(T \sim 10-20 \mathrm{~K})$ parental clouds (Pudritz 2002). The association with dense ambient material makes such objects best detectable as cold condensations at farinfrared and (sub)millimeter wavelengths. The short evolutionary timescales (Palla \& Stahler 1993) and low spatial density of massive objects require large-scale surveys for their identification. Many of the known intermediate- and high-mass protostellar candidates have therefore been discovered by followup studies towards IRAS sources (e.g. Shepherd et al. 2000; Cesaroni et al. 1997; Molinari et al. 1998; Beuther et al. 2002), which were selected on the basis of color and flux density criteria (e.g. by Wood \& Churchwell 1989; Palla et al. 1991).

The earliest stages of massive star formation are characterized by the initial conditions of their parental cloud cores with

Send offprint requests to: O. Krause,

e-mail: krause@mpia-hd.mpg.de

* Based on observations with the James-Clerk-Maxwell Telescope JCMT, the IRAM $30 \mathrm{~m}$ Telescope, the United Kingdom Infrared Telescope UKIRT, the MPIfR $100 \mathrm{~m}$ Telescope, the Calar Alto Observatory and the Infrared Space Observatory ISO, an ESA project funded by Member States (especially France, Germany, The Netherlands and the UK) and with the participation of ISAS and NASA. spectral energy distributions peaking beyond $100 \mu \mathrm{m}$ (Evans et al. 2002). In order to unveil such young objects we use the ISOPHOT (Lemke et al. 1996) $170 \mu \mathrm{m}$ Serendipity Survey (ISOSS) (Bogun et al. 1996), which is the largest high spatial resolution survey performed beyond the IRAS $100 \mu \mathrm{m}$ band. We selected bright and compact sources detected by ISOSS and IRAS with a flux ratio $F_{170 \mu \mathrm{m}} / F_{100 \mu \mathrm{m}}>2$, implying a low dust temperature $T<18 \mathrm{~K}$ and a large mass of the cold ISM in these objects. Since the clustered mode of massive star formation commonly involves young stellar objects of different evolutionary stages, we require the presence of embedded sources with thermal infrared excess as indicated by the 2MASS (Cutri et al. 2000) and MSX (Price et al. 2001) infrared surveys. The latter criterium also avoids confusion with cold interstellar cirrus. Here, we present the results of follow-up observations of the cold star-forming region ISOSS J 20298+3559 and show evidence for its early evolutionary stage.

\section{Observations}

\subsection{ISO far-infrared measurements}

$170 \mu \mathrm{m}$ data covering the region around ISOSS J 20298+3559 have been extracted from the ISOPHOT Serendipity Survey. The scans performed with the C200 $2 \times 2$ pixel detector array of stressed Ge:Ga with a pixel size of 89.4 arcsec provide a spatial resolution of 1.8 arcmin $F W H M$. A flatfield correction 


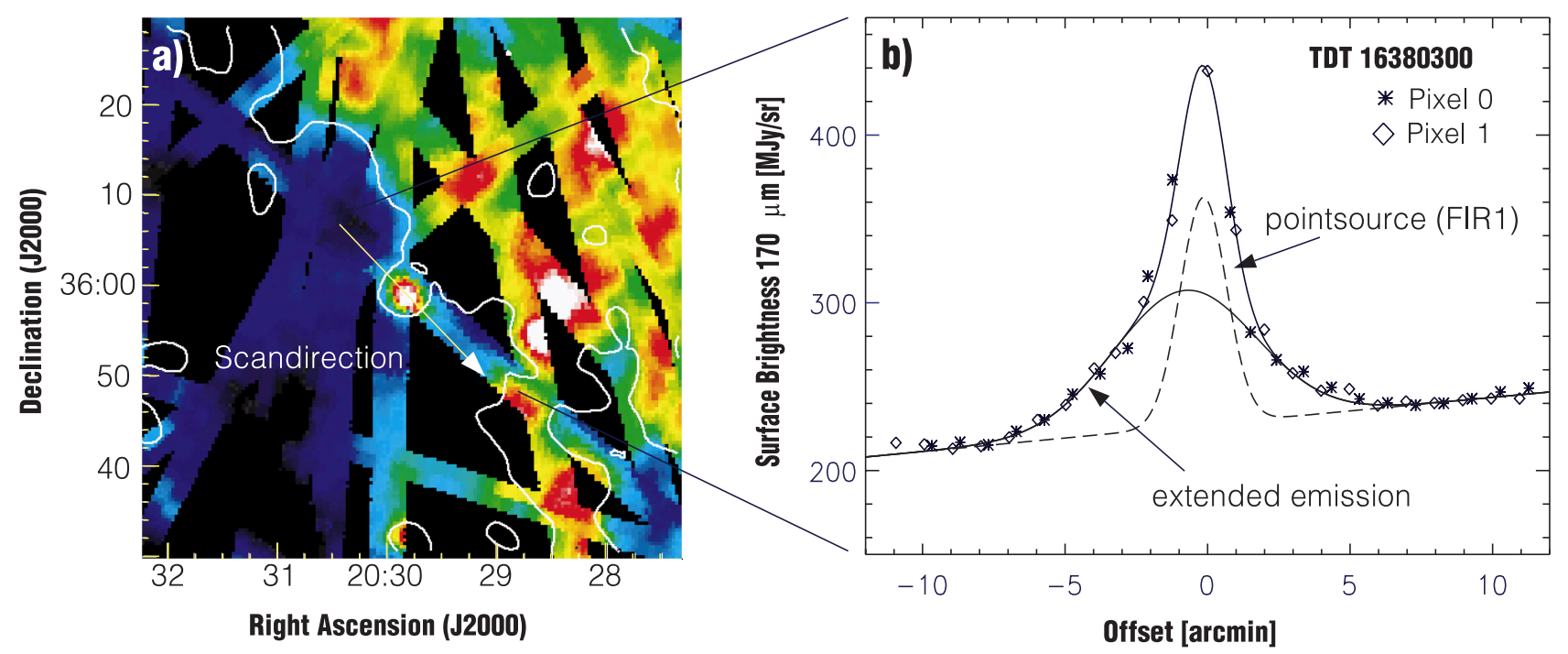

Fig. 1. a) $170 \mu \mathrm{m}$ continuum emission centered around ISOSS J 20298+3559, colors are from 180 to $400 \mathrm{MJy} / \mathrm{sr}$. Overlaid are IRAS $100 \mu \mathrm{m}$ contours from the HIRES processing (contour level $240 \mathrm{MJy} / \mathrm{sr}$ ). The map gives an impression of the typical sky coverage of the ISOPHOT Serendipity Survey towards the northern galactic plane. b) $170 \mu \mathrm{m}$ surface brightness along an ISOSS scan (TDT16380300) across the region (arrow in Fig. 1a), reconstructed from measurements with 2 pixels of the ISOPHOT C200 camera. The brightness profile has been decomposed into the contributions of a compact source (FIR1) and an extended component.
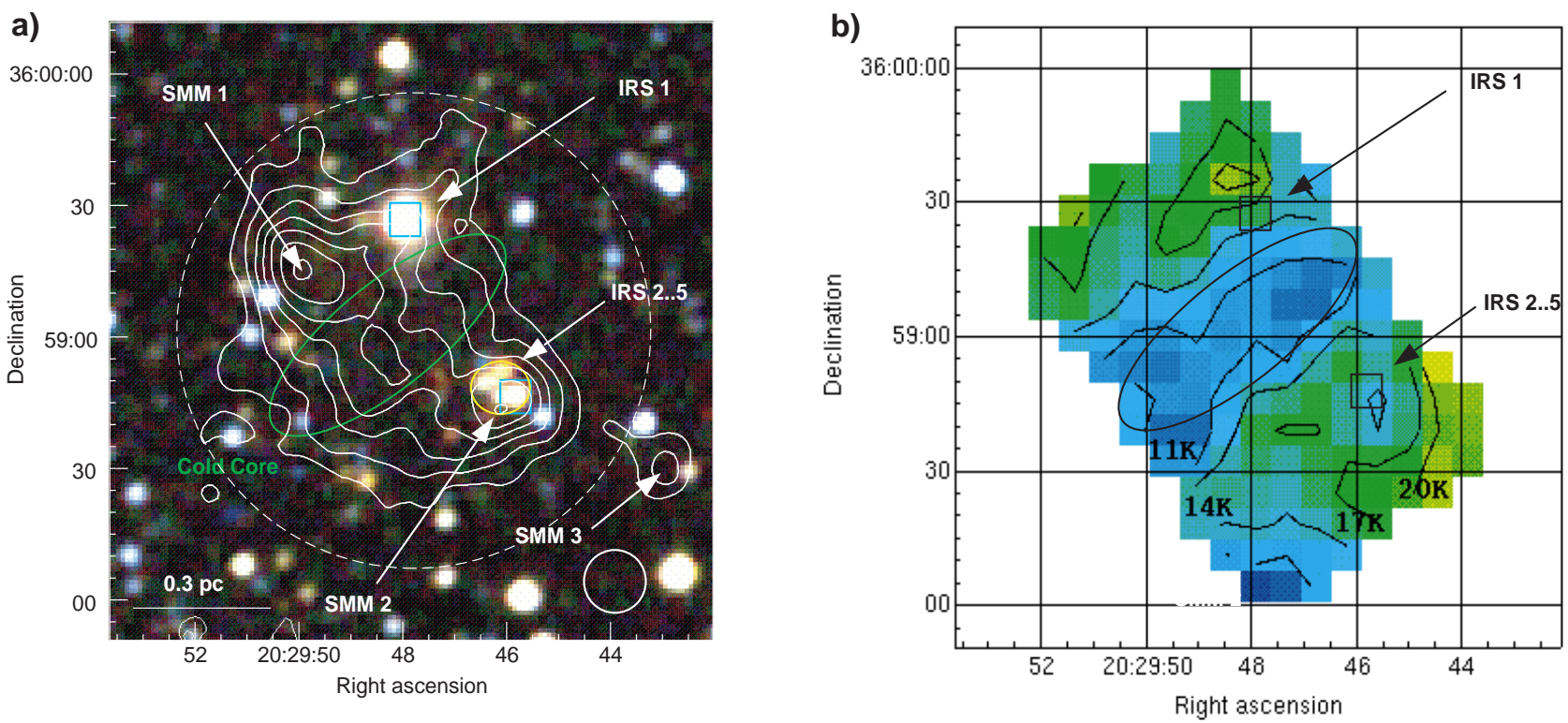

Fig. 2. a) $850 \mu \mathrm{m}$ continuum map of the compact ISOPHOT Serendipity Survey source FIR1, overlaid on a near-infrared $J H K_{\mathrm{S}}$-composite constructed from 2MASS data. Three compact dust condensations (SMM 1, SMM 2 and SMM 3) are detected, which are located in a diffuse extended emission. Mid-infrared sources detected by the MSX-satellite are marked with boxes. The submillimeter knot SMM 2 is associated with a small cluster of embedded NIR sources (IRS 2...5) as detected by the 2MASS and MSX surveys. IRS 1 was identified as a very young Herbig B2 star by our follow-up spectroscopy. The two compact submillimeter sources SMM1 \& SMM3 without any infrared counterparts are candidate Class 0 objects. The position of the very cold cloud core is indicated by an ellipse. Contour levels are starting at 67 and increasing by $33 \mathrm{mJy} / \mathrm{beam}$. The size of the SCUBA beam is indicated in the lower right, the dashed circle corresponds to the ISOPHOT beam. b) The dust color temperature distribution across FIR 1 shows the presence of a very cold $\left(T_{\mathrm{d}} \sim 11 \mathrm{~K}\right)$ core at the center of the cloud. The temperature profiles towards north-east and south-west indicate an external heating by the infrared sources IRS $1-5$. The temperature is calculated from the submillimeter spectral index between $450 \mu \mathrm{m}$ and $850 \mu \mathrm{m}$, assuming a dust emissivity $\beta=2$.

for the individual scans was derived from redundant measurements at scan crossings. The calibration accuracy is estimated to be $\pm 30 \%$ (Stickel et al. 2000)

\subsection{SCUBA \& MAMBO (sub)millimeter mapping}

Submillimeter jiggle maps at $450 \mu \mathrm{m}$ and $850 \mu \mathrm{m}$ were obtained with the SCUBA bolometer array (Holland et al. 1999) at the JCMT from Mauna Kea (Hawaii) on July 28, 2001 
under excellent and stable sky conditions $\left(\tau_{850 \mu \mathrm{m}}=0.185 \pm\right.$ 0.01 ). The observing time was $30 \mathrm{~min}$. The atmospheric transmission was determined from sky dips and water radiometer data (at JCMT and CSO). Mars, Uranus and CRL 618 served as calibrators. The data were reduced using the SCUBA User Reduction Facility (SURF) including identification of noisy bolometer pixels and removal of sky noise. The photometric accuracy derived from the calibration observations is $25 \%$ at $450 \mu \mathrm{m}$ and $20 \%$ at $850 \mu \mathrm{m}$. The measured $H P B W$ is 7.9 arc$\mathrm{sec}$ at $450 \mu \mathrm{m}$ and $14.9 \mathrm{arcsec}$ at $850 \mu \mathrm{m}$ (derived from observations of CRL618). In order to remove the error beam of the telescope, which contributes significantly to the $450 \mu \mathrm{m}$ data, the SCUBA maps were deconvolved with a beam map of Uranus using the SCLEAN algorithm (Keel 1991) and finally restored to the initial spatial resolution. We know that the maps may be distorted by chopping into ambient cloud emission. This will affect the $450 \mu \mathrm{m}$ flux more than the $850 \mu \mathrm{m}$ flux, because the error lobe is larger at $450 \mu \mathrm{m}$. The $450 \mu \mathrm{m}$ map was therefore finally corrected by adding a constant level of $5 \%$ of the peak value to the map, before convolving it to the final resolution.

$1.3 \mathrm{~mm}$ continuum observations were carried out on June 9 and June 11, 2001 with the IRAM $30 \mathrm{~m}$ telescope on Pico Veleta (Spain) using the 37-channel bolometer array MAMBO (Kreysa et al. 1998). Our observing strategy adopted On-Off measurements at 8 different raster positions in order to sample most of the object plane. The mode was chosen due to a rather high sky opacity of $\tau_{1.2 \mathrm{~mm}}=0.62$ and $\tau_{1.2 \mathrm{~mm}}=0.55$ and unstable atmosphere on the two days. Calibration measurements were obtained with the nearby source K30A. Data were reduced with the NIC package including sky noise reduction. The photometric accuracy is $30 \%$.

\subsection{UKIRT/MAX mid-infrared imaging}

Diffraction limited mid-infrared images were acquired with the MAX camera mounted at the $3.8 \mathrm{~m}$ United Kingdom Infrared Telescope UKIRT from Mauna Kea (Hawaii) on December 20, 2000. The MAX camera (Robberto et al. 1998) consists of a Rockwell $128 \times 128 \mathrm{Si}$ :As BIB array, providing a field of view of $35 \times 35 \operatorname{arcsec}^{2}$. Spectrophotometric data were obtained through three narrow band filters $\left(\lambda_{\mathrm{eff}} \sim 8.7,9.711 .6 \mu \mathrm{m}, \Delta \lambda \sim\right.$ $1.0 \mu \mathrm{m})$ and in the $M-, N$ - and $Q$-bands. The observations were made using standard beam switching techniques with a chop frequency of $2.5 \mathrm{~Hz}$ and a throw of $25 \operatorname{arcsec}\left(\mathrm{PA} 90^{\circ}\right) . \alpha$ Tau and $\alpha$ Boo served as standard stars, also for the narrow band filters where we derived synthetic photometry from data obtained by Cohen et al. (1996). The images were finally deconvolved by the algorithm from Richardson \& Lucy with standard stars as PSF estimators and have a resolution with a $F W H M$ of $\sim 0.6$ arcsec. The photometric accuracy is $20 \%$.

\subsection{Optical spectroscopy and photometry}

Optical long-slit spectroscopy was obtained using the TWIN double spectrograph at the $3.5 \mathrm{~m}$ telescope at Calar Alto (Spain) on December 12, 2000 with a spectral dispersion of $0.55 \AA /$ pixel for the blue $(3400-5500 \AA)$ and $1.1 \AA /$ pixel in the red (6300-7100 ̊) image channel. A photometric accuracy of $10 \%$ was obtained. The spectra were extracted according to the method of Horne (1986).

CCD images in the Johnson BVRI bands were obtained with the MPIA $0.7 \mathrm{~m}$ telescope on mount Königstuhl in Heidelberg (Germany), equipped with a Tektronix $1024 \times$ 1024 camera (scale $0.89 \mathrm{arcsec} / \mathrm{pixel})$. Data reduction of both spectroscopy and direct imaging was carried out using standard IRAF routines. I-band photometry of about 3000 stars has been derived using DAOPHOT (Stetson et al. 1987). The stellar photometry is complete to a limiting magnitude $I=18.8 \mathrm{mag}$ with a photometric uncertainty $\sigma<0.2 \mathrm{mag}$.

\subsection{Effelsberg $\mathrm{NH}_{3}$ measurements}

We carried out $\mathrm{NH}_{3}$ measurements with the MPIfR $100 \mathrm{~m}$ telescope at Effelsberg in April 2002. The HPBW at the observing frequency of $23.7 \mathrm{GHz}$ is $40^{\prime \prime}$. The facility $1.3 \mathrm{~cm}$ receiver was used with a typical system temperature (antenna temperature units) on the sky of $170 \mathrm{~K}$ in total power mode. The backend was the facility 8096 channel autocorrelator split into 4 bands in order to observe simultaneously both polarizations at the $\mathrm{NH}_{3}(1,1)$ and $(2,2)$ rest frequencies of $23694.495 \mathrm{MHz}$ and $23722.633 \mathrm{MHz}$, respectively. The resulting spectral resolution was $0.06 \mathrm{~km} \mathrm{~s}^{-1}$. Pointing and calibration were checked by continuum scans across NGC 7027 . We estimate a pointing accuracy of about $5^{\prime \prime}$. We calibrated our data assuming a main beam brightness temperature for NGC 7027 of $8.2 \mathrm{~K}$ corresponding to $5.86 \mathrm{Jy}$ (Baars et al. 1977). Our $\mathrm{NH}_{3}$ spectra were reduced using the GILDAS software, and analyzed following procedures described by Harju et al. (1993).

\section{Results}

\subsection{Morphology of the cold ISOSS source}

Figure 1 presents our far-infrared measurements of ISOSS J 20298+3559 obtained by the ISOPHOT Serendipity Survey. The source was detected by two individual scans of the survey. A $170 \mu \mathrm{m}$ continuum map constructed from all scans in the region (Fig. 1a) shows the compact object on a bright galactic background. Overlaid on the map are IRAS $100 \mu \mathrm{m}$ contours, indicating the previous detection of the associated source IRAS $20278+3549$. Due to the superior spatial resolution of ISOPHOT we could disentangle two emission components from a central scan across the object (Fig. 1b). The $170 \mu \mathrm{m}$ emission is well described by an unresolved source (FIR1) with a flux density of $F_{170 \mu \mathrm{m}}=110 \mathrm{Jy}$ and an extended emission component (size 5.9 arcmin $F W H M$, peak surface brightness $I_{170 \mu \mathrm{m}}=90 \mathrm{MJy} / \mathrm{sr}$ ).

High resolution sub-millimeter continuum maps at $450 \mu \mathrm{m}$ and $850 \mu \mathrm{m}$ with SCUBA and at $1.3 \mathrm{~mm}$ with MAMBO have been obtained towards the unresolved far-infrared source FIR1. The $850 \mu \mathrm{m}$ image superimposed on a near-infrared $\left(J H K_{\mathrm{S}}\right)$ color composite from the 2MASS survey is presented in Fig. 2. FIR1 indicated by the dashed circle is clearly resolved by the JCMT in the submillimeter. The map indicates the presence of a dense core (outlined by an ellipse) surrounded by extended 


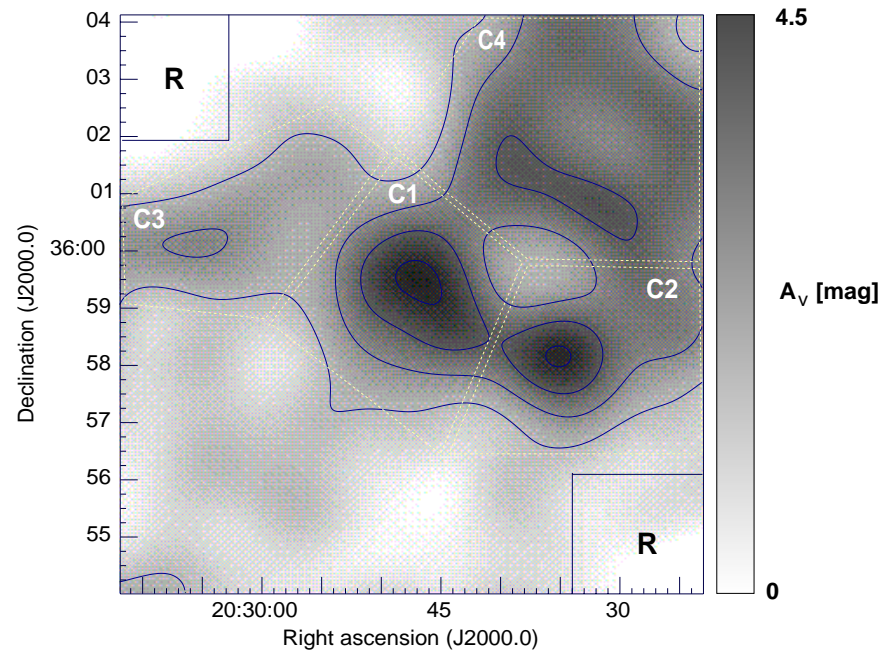

Fig. 3. Extinction map towards the star forming region ISOSS J 20298+3559 derived from $I$-band star counts and corrected for foreground stars. Four individual dark clouds (C1...C4) have been detected. Overlaid are contours of visual extinction $\mathrm{A}_{V}$ (contour levels at 1.5, 2.5, 3.5 and $4.5 \mathrm{mag}$ ). The rectangles marked with " $R$ " show the reference fields used to set the zero point of extinction. In order to obtain the total line-of-sight extinction, a foreground extinction of $A_{V}=2.7 \mathrm{mag}$ has to be added to the data.

emission, which covers an area of about $100 \times 70 \operatorname{arcsec}^{2}$ and accounts for the bulk of the flux. This extended emission is located northeast and southwest of the core and shows three compact dust condensations. We derived positions and fluxes of the compact sources by a two-dimensional Gaussian deconvolution using a local background estimation. The source sizes were determined from the $450 \mu \mathrm{m}$ data, which have the highest available resolution. The $1.2 \mathrm{~mm}$ fluxes of the fainter sources SMM 2 and SMM 3 could not be determined due to background confusion at that wavelength. The results are given in Table 1. The integrated (sub)millimeter flux density of FIR1 within a circular 2.4 arcmin aperture corresponding to the SCUBA field of view and covering most of the ISOPHOT beam are $F_{450 \mu \mathrm{m}}=27.5 \mathrm{Jy}, F_{850 \mu \mathrm{m}}=3.9 \mathrm{Jy}$ and $F_{1200 \mu \mathrm{m}}=1.1 \mathrm{Jy}$.

\subsection{Distance and structure of the cloud complex}

A well-known drawback for the use of kinematical distances towards Cygnus is the near-zero radial velocity gradient up to a distance of about $2 \mathrm{kpc}$ from the sun. Therefore we have created an extinction map to determine a reliable distance to ISOSS J 20298+3559 based on a Wolf diagram and to study the large-scale distribution of dust associated with the far-infrared source. We have obtained deep I-band images of the region using the MPIA $0.7 \mathrm{~m}$ telescope.

The extinction map shown in Fig. 3 was constructed according to the method of Bok (1956) and subsequently smoothed to the ISOPHOT resolution. An adaptive grid (reseau size $\sim 1$ arcmin) was used for optimization of the angular resolution with respect to the stellar density (Cambrésy et al. 1997). The observed cloud extinction $A^{\text {obs }}$ with respect to the reference positions was calculated from the number density $N^{b}$ of

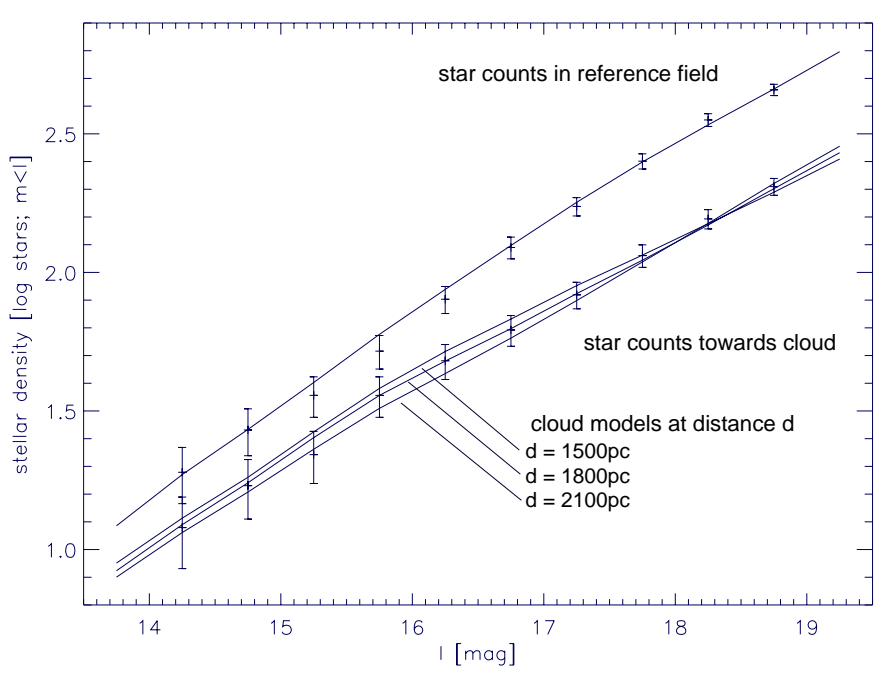

Fig. 4. Wolf-diagram of cumulative I-band star counts towards the dark cloud (lower points) and the reference fields (upper points). The error bars correspond to $\pm 1 \sigma$ of a Poisson statistics. The data are in good agreement with our predictions based on a Galactic stellar distribution model, indicated by the overlaid curves. The results of the Monte-Carlo simulations are consistent with a distance of $1.8 \pm$ $0.3 \mathrm{kpc}$ to the dark cloud complex.

stars seen towards the cloud and their unobscured reference density $N_{\text {ref }}^{b} / A^{\text {obs }}=-1 / a \log \left(N_{\text {ref }}^{b} / N^{b}\right)$, where $a$ denotes the slope of the $I$-band luminosity function. We use $A_{V}=2.07 A_{I}$ (Rieke \& Lebofsky 1985) to relate visual extinction to our I-band observations. The reference fields are marked with " $R$ " in Fig. 3 and are located in direction of the lowest dust column density as indicated by the $170 \mu \mathrm{m}$ continuum.

The extinction $A^{\text {obs }}$ derived from the simple Bok formula underestimates the true cloud extinction $A^{\text {true }}$ of a distant cloud due to the contamination by foreground stars. Foreground stars lower the density contrast towards the cloud. Since they are not affected by the cloud extinction, their number density remains constant: $N^{f}=N_{\text {ref }}^{f}$. In order to account for the stellar foreground and background population we apply the general form of the Bok formula (e.g. Cambrésy et al. 2002) providing the true extinction $A_{I}^{\text {true }}$ depending on the fraction of background stars.

Figure 4 presents the Wolf diagram for the reference field (upper curve) and towards the dense region $\left(A_{I}^{\mathrm{obs}}>1 \mathrm{mag}\right)$ of the cloud complex (lower curve). The logarithm of the cumulative distribution of apparent magnitudes is shown, error bars are $\pm 1 \sigma$ and are calculated according to Poisson statistics. The number density in the reference field agrees well with the prediction from the Galactic stellar distribution model from Robin \& Creeze et al. (1986), assuming an average interstellar extinction coefficient of $A_{V}=1.5 \mathrm{mag} \mathrm{kpc}^{-1}$ in the distance range $0<d<4 \mathrm{kpc}$ and $A_{V}=0.7 \mathrm{mag} \mathrm{kpc}^{-1}$ beyond $4 \mathrm{kpc}$. The higher extinction below $4 \mathrm{kpc}$ is attributed to dust in several giant molecular cloud complexes of the local spiral arm and extending out to the inter-arm gap between local and Perseus spiral arms at a distance of $4 \mathrm{kpc}$ (Ungerechts et al. 2000). The integrated $(0<d<5 \mathrm{kpc})$ line-of-sight extinction of $A_{V}=$ 6.7 mag towards the reference fields agrees with the value of 
Table 1. Photometry of (sub)millimeter sources associated with ISOSS J 20298+3559-FIR1.

\begin{tabular}{|c|c|c|c|c|c|c|c|c|c|}
\hline \multirow[t]{2}{*}{ Source } & \multirow[t]{2}{*}{$\begin{array}{c}\text { Position [J2000] } \\
\alpha\end{array}$} & \multirow[t]{2}{*}{$\delta$} & \multicolumn{3}{|c|}{$\begin{array}{c}\text { Total Flux } \\
\text { Jy }\end{array}$} & \multirow[t]{2}{*}{$\begin{array}{c}\text { Size } \\
\mathrm{pc}\end{array}$} & \multicolumn{3}{|c|}{$\begin{array}{c}\text { Peak Flux } \\
\text { mJy beam }^{-1}\end{array}$} \\
\hline & & & $450 \mu \mathrm{m}$ & $850 \mu \mathrm{m}$ & $1.2 \mathrm{~mm}$ & & $450 \mu \mathrm{m}$ & $850 \mu \mathrm{m}$ & $1.2 \mathrm{~mm}$ \\
\hline SMM 1 & $20: 29: 49.9$ & $+35: 59: 15$ & 1.26 & 0.233 & 0.075 & $0.1 \times 0.2$ & 690 & 251 & 80 \\
\hline SMM 2 & $20: 29: 45.6$ & $+35: 58: 46$ & 0.31 & 0.091 & - & $<0.07$ & 610 & 206 & - \\
\hline SMM 3 & $20: 29: 48.2$ & $+35: 59: 24$ & 1.05 & 0.155 & - & $0.15 \times 0.2$ & 290 & 117 & - \\
\hline cold core & $20: 29: 48.4$ & $+35: 58: 57$ & 6.02 & 0.902 & 0.33 & $0.55 \times 0.29$ & 410 & 203 & 60 \\
\hline extended & - & - & 18.86 & 2.47 & 0.7 & $0.9 \times 0.7$ & 368 & 173 & - \\
\hline
\end{tabular}

$A_{V}=6.9 \pm 0.7$ mag derived by Schlegel \& Finkbeiner (1998) from the IRAS $100 \mu \mathrm{m}$ dust continuum.

To interprete the shape of the Wolf diagram correctly, a model curve of an obscured region was determined from Monte-Carlo simulations using a Galactic stellar distribution model for the unobscured field star luminosity function. We assumed the extinction in the cloud to be Gaussian distributed for resolutions smaller than tested by the average extinction $\mathrm{A}_{I}^{\mathrm{obs}}$ of our integral aperture. The lower curves in Fig. 4 show the results of our simulations for three best-fit cloud models, which are consistent with our observed average extinction $\left(A_{I}^{\mathrm{obs}}=1.1 \mathrm{mag}\right)$ and are located at distances of 1.5, 1.8 and $2.1 \mathrm{kpc}$, respectively. From the observations we derive a cloud distance $d=1.8 \pm 0.3 \mathrm{kpc}$ and an average extinction of the cloud of $A_{V}=3.1 \pm 0.5 \mathrm{mag}$, where the errors mainly originate from the Poisson statistics of our star counts. Using the interstellar extinction coefficient of $A_{V}=1.5 \mathrm{mag} \mathrm{kpc}^{-1}$ determined from the reference field, the foreground extinction towards ISOSS J $20298+3559$ is $A_{V}=2.7 \pm 0.3 \mathrm{mag}$.

We compared our result with the empirical distance estimator $d=320 N^{0.57}$ pc by Herbst \& Saywer (1987), which relates the cloud distance to the number $N$ of foreground stars counted within a circular aperture of 5 arcmin diameter on the POSS blue print. Using $B$-band star counts from the USNOPMM catalogue (Monet 1996) we obtain a distance of $d \sim$ $2.2 \mathrm{kpc}$ for the cloud complex. The formula is however only valid for opaque dark clouds $\left(A_{V}>7 \mathrm{mag}\right)$ and the higher distance might originate in the partly translucent character of ISOSS J 20298+3559.

\subsection{Cloud masses}

The extinction map clearly reveals the presence of a dusty cloud complex with 4 different dark clouds (C1...C4). C1 coincides with the cold source FIR1 and accounts for the extended far-infrared emission shown in Fig. 1b. Despite being covered by our $170 \mu \mathrm{m}$ scan, the south-western cloud C2 does not show up with a strong $170 \mu \mathrm{m}$ excess and we find an upper limit of $F_{170 \mu \mathrm{m}}<15 \mathrm{Jy}$. The cloud complex also shows a small extinction ridge towards the east (C3) and extends to the northwest (C4).

Cloud masses were derived from the extinction map, using the relation by Dickman (1978):

$M=(\Delta \Omega d)^{2} \mu \frac{N_{\mathrm{H}}}{A_{V}} \sum_{i} A_{V}(i)$,
Table 2. Individual clouds towards ISOSS J 20298+3559. The columns are: (1) cloud, (2) position of peak extinction $A_{V}$, (3) mass $M_{\mathrm{HI}}+M_{\mathrm{H}_{2}}$, (4) peak extinction $A_{V}$ and (5) size.

\begin{tabular}{lcccc}
\hline \hline Source & $\begin{array}{c}\text { Position [J2000] } \\
\alpha, \delta\end{array}$ & $\begin{array}{c}\text { Mass } \\
M_{\odot}\end{array}$ & $\begin{array}{c}\text { Peak } A_{V} \\
\text { mag }\end{array}$ & $\begin{array}{c}\text { Size } \\
\mathrm{pc}^{2}\end{array}$ \\
\hline $\mathrm{C} 1$ & $20: 29: 46.8+35: 59: 30$ & 188 & 4.7 & $2.0 \times 1.7$ \\
$\mathrm{C} 2$ & $20: 29: 35.1+35: 58: 09$ & 162 & 4.6 & $1.9 \times 1.7$ \\
$\mathrm{C} 3$ & $20: 30: 05.3+36: 00: 07$ & 115 & 2.6 & $2.5 \times 1.5$ \\
$\mathrm{C} 4$ & $20: 29: 31.9+36: 00: 49$ & 295 & 3.7 & $2.3 \times 2.2$ \\
\hline
\end{tabular}

where $\Delta \Omega$ is the angular size of the map, $\mathrm{d}$ is the distance to the cloud, $\mu$ is the mean molecular weight corrected for helium abundance, and $i$ corresponds to a map pixel. With the dust-to-gas ratio proposed by Savage \& Mathis (1979), $N_{\mathrm{H}} / A_{V}=1.87 \times 10^{21} \mathrm{~cm}^{-2} \mathrm{mag}^{-1}\left(N_{\mathrm{H}}=N_{\mathrm{HI}}+2 N_{\mathrm{H}_{2}}\right)$ we obtain a total mass of the cloud complex of $760 M_{\odot}$. We list in Table 2 parameters of the four individual clouds. Due to systematic uncertainties in the extinction values and the conversion factor, we assume the mass estimates to be accurate within a factor of 2 .

\subsection{Dust properties of FIR1}

Figure 5 displays the spectral energy distribution (SED) for the cold region FIR 1. The spectral energy distribution longwards of $100 \mu \mathrm{m}$ is dominated by large grains, which are at an equilibrium temperature within the surrounding radiation field.

The flux density of the diffuse dust component in FIR1 (excluding the knots SMM 1, SMM 2 and SMM 3) is well characterized by optically thin thermal radiation of two modified blackbodies $F_{v} \propto v^{\beta} B\left(v, T_{\mathrm{d}}\right)$ with a dust emissivity $\beta=2$ and dust temperatures $T_{\mathrm{d}}=13 \pm 2 \mathrm{~K}$ and $T_{\mathrm{d}}=19 \pm 2 \mathrm{~K}$. As can be seen from Table 1, the contribution of SMM 1, SMM 2 and SMM 3 to the total (sub)millimeter flux is less than $15 \%$. In the large beam measurements of the IRAS/ISO satellites we cannot separate these sources. If they contributed significantly more FIR flux than estimated from our submillimeter data, the dust temperatures of the diffuse dust component would be even lower and the resulting dust mass higher.

Drawing the conclusion of a very cold dust component merely from large-beam integrated photometry is critical without any radiative transfer model. Our spatially resolved submillimeter data allow, however, to confirm the presence of a 
Table 3. Results of the ammonia observations of the cold core region. The columns are (1) line, (2) main beam brightness temperature of the maingroup, (3) $v_{\mathrm{LSR}}$, (4) FWHM from fit, (5) main group line area, (6) total line area of satellite groups and (7) $\tau_{\text {main }}$.

\begin{tabular}{ccccccc}
\hline \hline Line & $\begin{array}{c}T_{\mathrm{MB}}(\mathrm{main}) \\
\mathrm{K}\end{array}$ & $\begin{array}{c}v_{\mathrm{LSR}} \\
\mathrm{km} \mathrm{s}^{-1}\end{array}$ & $\begin{array}{c}F W H M \\
\mathrm{~km} \mathrm{~s}^{-1}\end{array}$ & $\begin{array}{c}\text { areamain } \\
\mathrm{K} \mathrm{km} \mathrm{s}^{-1}\end{array}$ & $\begin{array}{c}\text { areasatel } \\
\mathrm{K} \mathrm{km} \mathrm{s}^{-1}\end{array}$ & $\tau$ \\
\hline$(2,2)$ & $0.139(0.074)$ & $0.66(0.13)$ & $0.883(0.408)$ & 0.146 & & $0.78(0.10)$ \\
$(1,1)$ & $0.329(0.076)$ & $0.58(0.02)$ & $0.955(0.065)$ & 0.34 & 0.19 & $0.10(0.05)$ \\
\hline
\end{tabular}

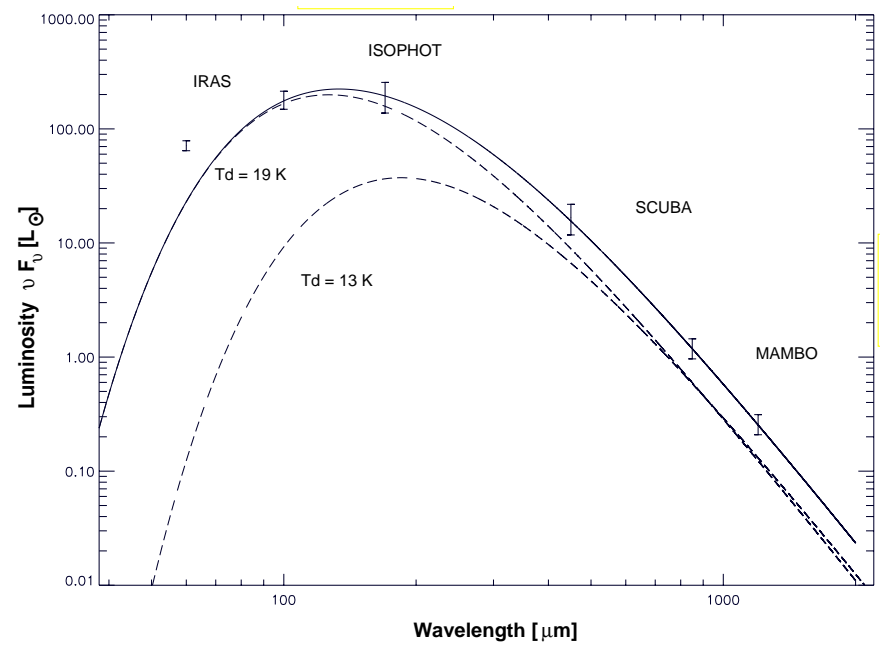

Fig. 5. Spectral energy distribution of the cold ISOSS source FIR1. The total flux density is well characterized by the superposition of optically thin thermal radiation from two modified blackbodies $(\beta=2)$ with dust temperatures of $T_{\mathrm{d}}=13 \pm 2 \mathrm{~K}$ and $T_{\mathrm{d}}=19 \pm 2 \mathrm{~K}$, respectively.

very cold dust component from the dust color temperature map of ISOSS J 20298+3559-FIR1 presented in Fig. 2b. The map was obtained from the deconvolved $450 \mu \mathrm{m}$ and $850 \mu \mathrm{m}$ maps smoothed to 14.9 arcsec resolution. The color temperature $T_{\text {col }}$ was derived from the spectral index between the two wavelengths and is given by

$F_{v_{1}} / F_{v_{2}}=v_{1}^{3+\beta}\left(\mathrm{e}^{h v_{2} / k T_{\text {col }}}-1\right) / v_{2}^{3+\beta}\left(\mathrm{e}^{h v_{1} / k T_{\text {col }}}-1\right)$,

with a dust emissivity $\beta=2$. A decreasing color temperature and a clear $850 \mu \mathrm{m}$ (and $1.2 \mathrm{~mm}$ ) excess is observed towards the cloud center, exhibiting a minimum temperature of only $T_{\text {col }}=11 \pm 2 \mathrm{~K}$.

Optical depth effects in the sub-millimeter or a change in the dust emissivity $(\beta<2)$ could affect the spectral index. We conclude, however, that these effects are small and the dust temperature dominates the spectral index: the peak column density towards the cloud core derived from our $850 \mu \mathrm{m}$ data is $N\left(\mathrm{H}_{2}\right)=2.2 \times 10^{22} \mathrm{~cm}^{-2}$, in good agreement with our ammonia observation (see next section). The corresponding optical depth is $\tau_{850}=2 \times 10^{-3} \ll 1$. Assuming a roughly ellipsoidal core geometry of $0.55 \times 0.29 \mathrm{pc}^{2}$ as indicated in Fig. 2 we obtain an absolute molecular gas density of $N\left(\mathrm{H}_{2}\right)=$ $2 \times 10^{5} \mathrm{~cm}^{-3}$. Concerning $\beta$, the centrally decreasing temperature profile points to an external heating of the core region. Therefore the conditions in our core region basically resemble

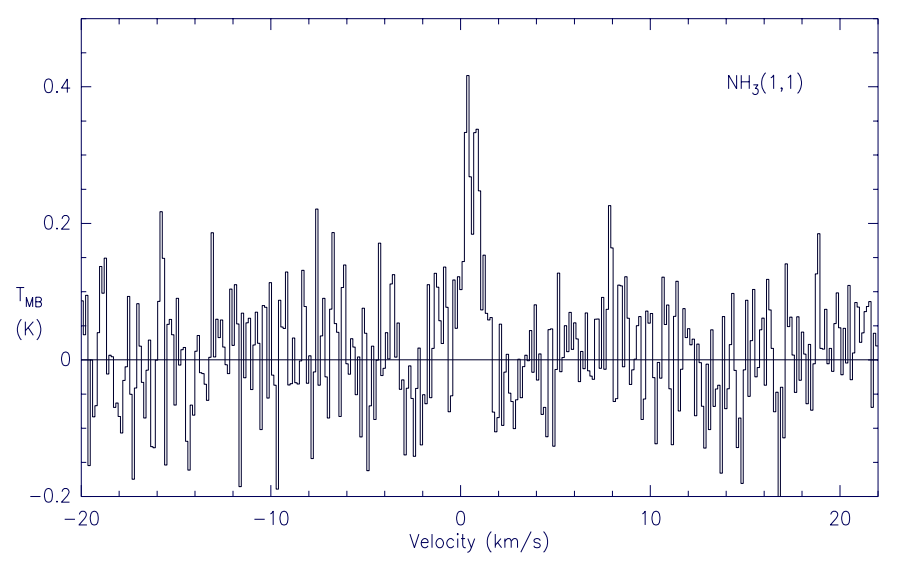

Fig. 6. Effelsberg $\mathrm{NH}_{3}$ spectrum taken at the position of the cold core region (Table 1) in ISOSS J 20298+3559-FIR1.

observations of nearby very cold pre-stellar cores for which $\beta=2$ was found (e.g. Juvela et al. 2001; Ward-Thompson et al. 2002).

Masses for the emitting regions have been derived from the optically thin emission in the submillimeter. The dust mass is given by $M_{\mathrm{d}}=F_{850} d^{2} /\left(\kappa_{850} B_{850}\left(T_{\mathrm{d}}\right)\right)$, where we used a dust mass absorption coefficient of $\kappa_{850}=2.1 \mathrm{~cm}^{2} \mathrm{~g}^{-1}$ following Ossenkopf \& Henning (1997) for dense protostellar cores. Following Sodroski et al. (1997) we assume a gas-to-dust mass ratio of 150 and derive a total gas mass $M_{\mathrm{H}_{2}}=120 M_{\odot} \pm 24 M_{\odot}$ for FIR1. The total mass contained in the cold core is $M_{\mathrm{H}_{2}}=$ $50 \pm 10 M_{\odot}$.

We calculated the bolometric FIR luminosities re-radiated from large grains by integrating over the Planckian radiation and find for the region $L_{\mathrm{FIR}}=250 L_{\odot}$ and for the cold core $L_{\mathrm{FIR}}=15 L_{\odot}$, respectively.

\subsection{Dense gas in the cold core region}

It is important to verify the physical conditions derived from our dust continuum observations by molecular line observations, which reflect the physical conditions of the gas phase ISM. $\mathrm{NH}_{3}$ is a very useful indicator of molecular cloud temperatures and column densities (e.g., Walmsley \& Ungerechts 1983).

We detected the $\mathrm{NH}_{3}(1,1)$ line with a $S / N$ of 4 and the $\mathrm{NH}_{3}(2,2)$ line with a $S / N$ of 2 . Figure 6 presents the $(1,1)$ spectrum. We derived the $\mathrm{NH}_{3}(1,1)$ line optical depth $\tau_{11}=0.10 \pm 0.05$ and estimated the $(1,1)(2,2)$ rotational temperature $T_{12}=19.2 \pm 4.0 \mathrm{~K}$ and the gas kinetic 
a)

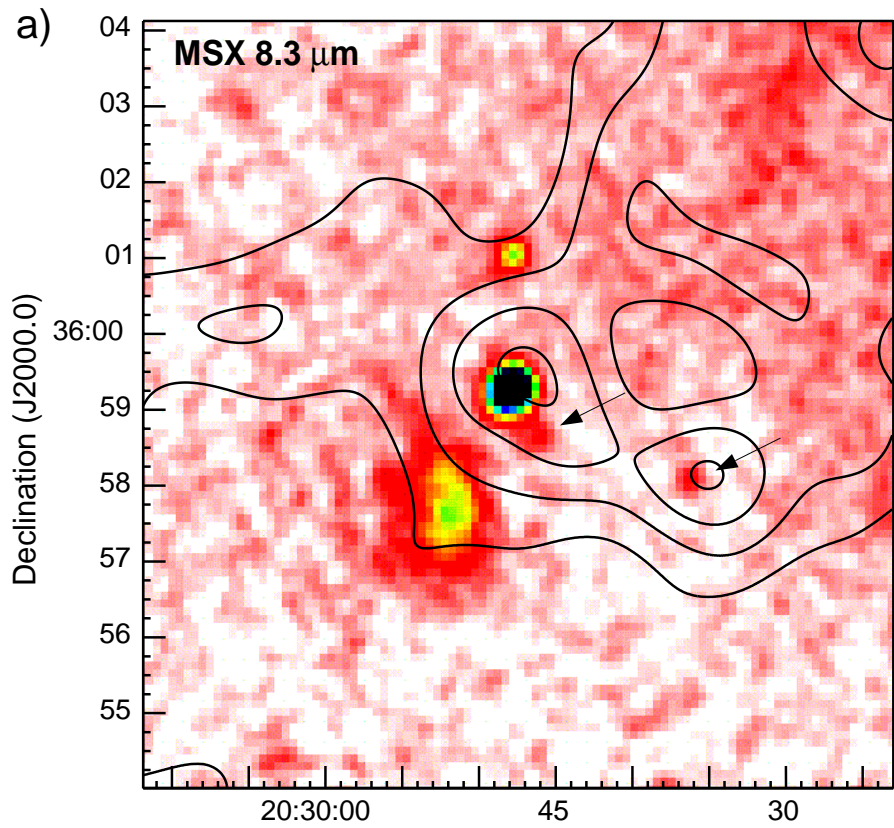

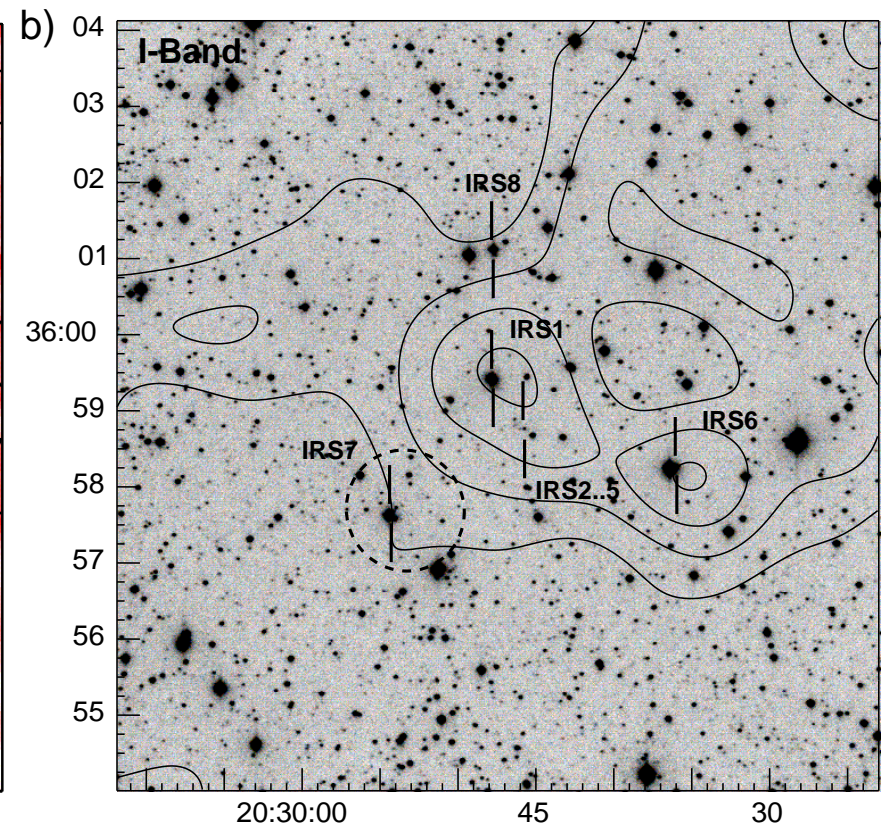

Right ascension (J2000.0)

Fig. 7. a) $8.3 \mu \mathrm{m}$ image obtained by the MSX satellite with contours of visual extinction overlaid (see Fig. 3 , contour levels $A_{V}=1.5,2.5,3.5$ and $4.5 \mathrm{mag}$ ). Colors are stretching from 5 to $40 \mathrm{mJy} \mathrm{pixel}^{-1}$. Five sources are detected by MSX above the $5 \sigma$ level, the two fainter ones are marked by arrows. Note the extended source towards the south-east, which shows an external illumination of the outer rim of the molecular cloud. The illuminating star is IRS7. b) $I$-band image of the ISOSS J 20298+3559 region, obtained with the MPIA 0.7 m telescope with contours of visual extinction overlaid. The optical identifications of mid-infrared sources seen by the MSX satellite are labeled.

temperature $T_{\text {kin }}=21.3 \pm 5.0 \mathrm{~K}$. The derived ammonia column density was estimated as $N\left(\mathrm{NH}_{3}\right)=1.32 \pm 0.24 \times 10^{14} \mathrm{~cm}^{-2}$. From the $N\left(\mathrm{H}_{2}\right)$ column density obtained by our submillimeter continuum observations and smoothed to 40 arcsec resolution we derive a molecular hydrogen column density of $N\left(\mathrm{H}_{2}\right)=1.3 \pm 0.6 \times 10^{22} \mathrm{~cm}^{-2}$ for the region covered by the Effelsberg beam. This corresponds to a fractional abundance of $\chi\left(\mathrm{NH}_{3}\right)=N\left(\mathrm{NH}_{3}\right) / N\left(\mathrm{H}_{2}\right)=1.0 \times 10^{-8}$ which is in the range found by Molinari et al. (2000) in their study of ammonia clumps associated with young intermediate- and highmass star forming regions. Ammonia in ISOSS J 20298+3559 seems however to be under-abundant when comparing to the values given by Harju et al. (1993). They find a mean value of $\chi\left(\mathrm{NH}_{3}\right) \sim 2.6 \times 10^{-8}$ in a sample of 22 ammonia clumps in Orion. Ammonia is considered as a molecule characterizing later stages of chemical evolution. Myers \& Benson (1993) found $\mathrm{NH}_{3}$ to be more abundant in older cores, where stars have already formed. This may indicate that ISOSS J 20298+3559 is a rather young object. Table 3 summarizes our ammonia results.

ISOSS J $20298+3559$ is located towards the southern outskirts of the Cygnus X Giant Molecular Cloud (Dame \& Thaddeus 1985). The majority of active and luminous star forming regions in this giant molecular cloud is found at distances between 1.2 and $2.5 \mathrm{kpc}$ (Odenwald \& Schwartz 1993), corresponding to radial velocities (LSR) between $-10 \mathrm{~km} \mathrm{~s}^{-1}$ and $+10 \mathrm{~km} \mathrm{~s}^{-1}$. The ammonia line velocity $v_{\mathrm{LSR}}=0.58 \pm$ $0.06 \mathrm{~km} \mathrm{~s}^{-1}$ is consistent with the kinematical association with the Cygnus X GMC.

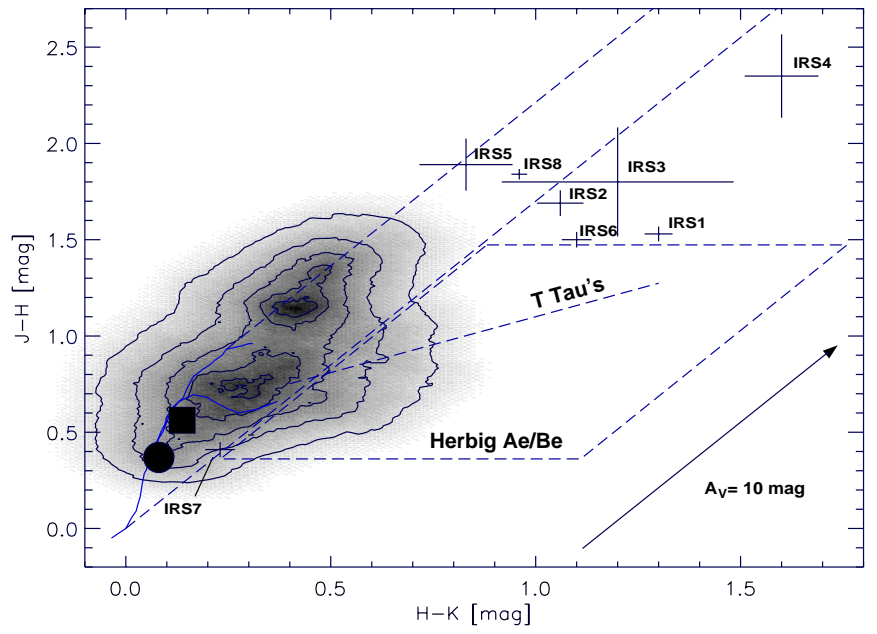

Fig. 8. $2 \mathrm{MASS} J H K_{\mathrm{S}}$ color-color diagram towards the cloud complex. The number density of field stars is indicated by contours. The solid curve in the lower left of the diagram is the locus of unreddened main sequence stars and giants, their reddening band is indicated between the two dashed lines. Several of the MSX counterparts IRS1...8 show an infrared excess characteristic for young stellar objects, as indicated by the colors of Herbig $\mathrm{Ae} / \mathrm{Be}$ and $\mathrm{T}$ Tauri stars. Also given is the redenning vector from Rieke \& Lebofsky (1985).

\subsection{Young stellar objects in the cloud complex}

Mid-infrared observations are well suited to identify young stellar objects enshrouded by warm circumstellar dust. An MSX $8.3 \mu \mathrm{m}$ image covering the region of our extinction map 
Table 4. Infrared sources associated with the cloud complex. The columns are: (1) IR source label, (2) position (3-6) near-infrared brightness in $I J H K_{\mathrm{S}}$ bands, (7) $8.3 \mu \mathrm{m}$ flux, (8) 2MASS PSC identification, (9) additional remarks.

\begin{tabular}{|c|c|c|c|c|c|c|c|c|}
\hline Source & $\begin{array}{c}\text { Position }[\mathrm{J} 2000] \\
\alpha, \delta\end{array}$ & \multicolumn{4}{|c|}{ mag } & $\begin{array}{c}8.3 \mu \mathrm{m} \\
\mathrm{mJy}\end{array}$ & $\begin{array}{c}\text { 2MASS } \\
\text { counterpart }\end{array}$ & remarks \\
\hline IRS 1 & $20: 29: 47.9+35: 59: 26$ & 12.90 & 10.98 & 9.45 & 8.15 & 1740 & J $2029479+355926$ & Herbig B2 star \\
\hline IRS 2 & $20: 29: 45.8+35: 58: 47$ & 17.23 & 14.70 & 13.01 & 11.95 & 90 & J $2029458+355847$ & brightest member of cluster IRS $2 \ldots 5$ \\
\hline IRS 3 & $20: 29: 46.1+35: 58: 46$ & 18.54 & $16.1^{\star}$ & $14.3^{\star}$ & $13.1^{\star}$ & | & & $J H K_{\mathrm{S}}$ fluxes from $2 \mathrm{MASS}$ map \\
\hline IRS 4 & $20: 29: 46.1+36: 58: 52$ & 19.5 & $16.2^{\star}$ & 13.85 & 12.25 & | & J $2029461+365852$ & $J$ flux from 2 MASS map \\
\hline IRS 5 & $20: 29: 46.4+35: 58: 50$ & 18.44 & 15.74 & 13.85 & 13.02 & | & J $2029464+355850$ & \\
\hline IRS 6 & $20: 29: 36.0+35: 58: 18$ & 15.77 & 13.33 & 11.83 & 10.73 & 75 & J $2029360+355818$ & \\
\hline IRS 7 & $20: 29: 54.5+35: 57: 38$ & 12.48 & 11.38 & 10.97 & 10.74 & 1870 & J $2029545+355738$ & MIR nebula + illuminating star \\
\hline IRS 8 & $20: 29: 47.9+36: 01: 08$ & 13.09 & 9.78 & 7.94 & 6.98 & 225 & J $2029479+360108$ & background star \\
\hline
\end{tabular}

is presented in Fig. 7a. Four compact and one extended midinfrared sources have been detected above the $5 \sigma$ noise level $(\sigma \sim 15 \mathrm{mJy} /$ beam). The astrometric accuracy of the MSX image ( $\sim 2$ arcsec rms) allows the cross-identification with objects from our $I$-band image shown in Fig. $7 \mathrm{~b}$ and the 2MASS point source catalogue. All four compact MSX sources coincide in position with very red $\left(I-K_{\mathrm{S}}=5 . .7 \mathrm{mag}\right)$ near-infrared counterparts. The objects are marked in Figs. 2 and $7 \mathrm{~b}$. Astrometry and photometry of the sources are given in Table 4, where the $8.3 \mu \mathrm{m}$ fluxes were derived from the MSX map performing aperture photometry, yielding a photometric accuracy of $25 \%$.

The intrinsic excess emission and extinction of the MSX near-infrared counterparts are characterized by the $(J-H)$ vs. $\left(H-K_{\mathrm{S}}\right)$ color-color diagram presented in Fig. 8, where the colors of IRS $1 \ldots 8$ are plotted with their photometric $1 \sigma$ errors. The solid curve corresponds to the loci of unreddened main sequence and giant branch stars (Bessel \& Brett 1988) and is extended by the reddening band, which confines stars with normal photospheres (dashed parallel lines).

IRS 1, IRS 2, IRS 3, IRS 4, and IRS 6 lie to the right side and show an intrinsic infrared excess due to warm circumstellar dust. Their observed colors are consistent with reddened $\left(A_{V} \lesssim\right.$ $15 \mathrm{mag}$ ) pre-main-sequence stars of low- to intermediate-mass as indicated in the diagram by the loci of unreddened Herbig Ae/Be (dashed parallelogram, Hillenbrand et al. 1992) and T Tauri stars (dashed line, Meyer et al. 1997). The derived extinction is smaller than the total line-of-sight extinction derived from our $850 \mu \mathrm{m}$, yielding $A_{V}=18 \mathrm{mag}$ towards IRS 1 and $A_{V}=23$ mag towards the small cluster IRS 2, IRS 3, IRS 4 and IRS 5. The sources are not affected by the total dust column, and are therefore either foreground objects or embedded in the cloud complex. The positional coincidence of dust with IRS 1 ...5 (Fig. 2) favors however the association with the dark cloud: IRS 1 is located on a dust ridge in the northwest of the central region of the cloud $\mathrm{C} 1$, IRS 2 coincides in position with the compact submillimeter source SMM 2 and is surrounded by a compact cluster of a least 3 further red sources.

IRS 5, IRS 7 and IRS 8 show no infrared excess. IRS 5 and IRS 8 are reddened with about $\left(A_{V} \sim 15 \mathrm{mag}\right)$. The corresponding line-of-sight extinctions from our extinction maps are 23 mag for IRS 5 and 4.5 mag for IRS 7 and IRS 8. which are located at the border of the cloud. This suggests that also
IRS 5 and IRS 7 are associated with the cloud, while IRS 8 is a background giant.

IRS 7 is located approximately in the center of a spherically segmented mid-infrared nebula seen in Fig. 7a. The nebula is located in the south-east of the dark cloud $\mathrm{C} 1$ and indicates an external illumination of the outer rim of the molecular cloud by IRS 7 . The nebula and IRS 7 coincide in position with the source IRAS $20279+3547$. The flux ratio $F_{12 \mu \mathrm{m}} / F_{25 \mu \mathrm{m}}>1.4$ and the strong emission in the MSX $8.3 \mu \mathrm{m}$ band, which contains a strong PAH feature, indicate a reflection nebula around a B type star. In order to clarify whether IRS 7 is the illuminating source we have obtained $B V R I$ photometry of the source. Comparing with the colors of main sequence stars we obtain a best fit for $A_{V}=4.2 \pm 0.3$ mag and a spectral type B4...B6, in agreement with the total line-of-sight extinction $A_{V}=4.2 \pm$ 0.5 mag towards IRS 7 from our extinction map.

\subsection{The embedded Herbig B2 star IRS 1}

In order to establish the pre-main-sequence nature of the bright source IRS 1 we have obtained optical long-slit spectra presented in Fig. 9. The spectra display strong emission lines of HI, Fe II, N I, Fe I which are all characteristic for luminous young stellar objects (Cohen \& Kuhui 1979). Parameters of important spectral features are given in Table 5.

We have several strong indications that IRS 1 is a rare example of an early B type pre-main-sequence object: He I absorption lines of presumably photospheric origin have been detected at $6678 \AA$ and $7065 \AA$. Comparing their strength with the theoretical values for stellar atmospheres given by Auer \& Mihalas (1973) we derive a spectral type of B2 $\left(T_{\text {eff }}=\right.$ $20000 \pm 2500 \mathrm{~K}, \log g=4.0$ ). This is consistent with the presence of NI emission lines which are confined to hotter Be type stars due to their high-ionization energy $(14.5 \mathrm{eV})$ and the large line excitation $(\sim 10 \mathrm{eV})$ (Andrillat et al. 1988). The relative strength of FeII with respect to neutral FeI also requires the presence of a more ionized line-emitting region than associated with classical T Tauri stars (Hamann \& Persson 1992), where FeI lines often rival the strength of FeII. In order to derive the rotational velocity of IRS 1 from the He I lines we have used the rotational broadening function given by Gray (1976). We obtain $v \sin (i)=158 \pm 25 \mathrm{~km} \mathrm{~s}^{-1}$, in agreement with a premain-sequence star of intermediate mass (Finkenzeller 1985). 


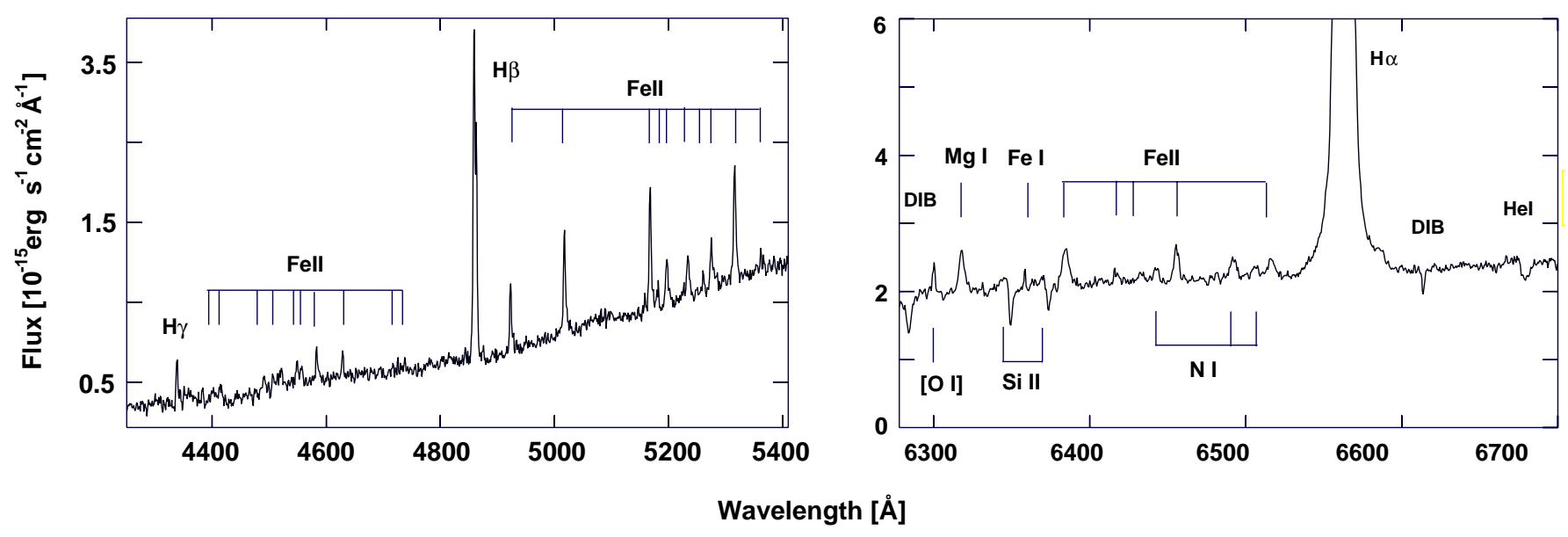

Fig. 9. Optical spectrum of the YSO ISOSS J 20298+3559-IRS 1 taken with the TWIN spectrograph at the $3.5 \mathrm{~m}$ telescope at Calar Alto. A spectral type B2 was inferred from photospheric He I absorption features. The spectrum is dominated by intense emission lines. They originate in a dense emission region, indicated by Fe II emission.

Table 5. Observed strong features in the optical spectrum of ISOSS J 20298+3559-IRS1. The columns are: (1) transition, (2) observed peak wavelength, (3) equivalent width, (4) line flux.

\begin{tabular}{lccc}
\hline \hline Line & $\begin{array}{c}\text { Obs. Wavelength } \\
{[\AA]}\end{array}$ & $\begin{array}{c}E W \\
{[\AA]}\end{array}$ & $\begin{array}{c}\text { Flux } \\
{\left[10^{-16} \mathrm{erg} \mathrm{cm}^{-2} \mathrm{~s}^{-1}\right]}\end{array}$ \\
\hline $\mathrm{H} \alpha$ & 6562.8 & -200 & 1950 \\
$\mathrm{H} \beta$ & 4861.4 & -19.9 & 99.8 \\
$\mathrm{H} \gamma$ & 4340.4 & -5.2 & 15.2 \\
$\mathrm{HeI}$ & 6678.15 & 0.55 & -13.8 \\
$\mathrm{HeI}$ & 7065.18 & 0.29 & -8.5 \\
$\mathrm{FeII}$ & 4923.06 & -2.8 & 19.2 \\
$\mathrm{FeII}$ & 5017.36 & -3.5 & 19.2 \\
$\mathrm{FeII}$ & 5316.05 & -4.3 & 48.3 \\
$\mathrm{FeII}$ & 5167.75 & -3.4 & 33.4 \\
$\mathrm{FeI}$ & 6358.56 & -0.2 & 4.8 \\
$\mathrm{MgI}(+\mathrm{FeI})$ & 6317.84 & -1.3 & 26.8 \\
$\mathrm{DIB}$ & 6613.81 & 0.3 & -6.5 \\
$\mathrm{NI}$ & 6442.02 & -0.4 & 9.4 \\
$\mathrm{NI}$ & 6491.36 & -0.8 & 16.9 \\
$\mathrm{NI}$ & 6505.58 & -0.8 & 18.1 \\
\hline
\end{tabular}

A small nebulosity (size $15 \times 8 \operatorname{arcsec}^{2}, \mathrm{PA}=40^{\circ}$ ) around IRS 1 which is visible on POSS- $B$ and $-R$ plates was covered by our long slit spectra and identified as a reflection nebula. Its presence and the association with the dark cloud $\mathrm{C} 1$ make the emission line object IRS1 by definition a Herbig Be star (Herbig 1960). According to the most comprehensive catalogue of Herbig Ae/Be stars compiled by Thé et al. (1994), only 20 objects known to date have a spectral type B2 or earlier.

IRS 1 (=MSX5C_G075.5314-01.8159) was detected by MSX at 8.3, 12.1 and $14.7 \mu \mathrm{m}$ and is included in the MSX point source catalogue (Egan et al. 1999). The source is unresolved by the small MSX telescope (beam $\sim 12$ arcsec). In order to explore the nature of the thermal excess emission of warm dust around IRS 1 we have obtained diffraction-limited subarcsecond images with the thermal infrared camera MAX at UKIRT. The observations cover the $M, N$ and $Q$ bands as well
Table 6. Optical and mid-infrared photometry of ISOSS J 20298+3549-IRS1 given in magnitudes.

\begin{tabular}{lccccccccc}
\hline \hline Band & $B$ & $V$ & $R$ & $M$ & $P 8$ & $S 9$ & $N$ & $P 11$ & $Q$ \\
\hline Flux & 17.3 & 15.7 & 14.5 & 5.7 & 3.9 & 4.1 & 3.7 & 3.1 & 2.6 \\
$\sigma$ & 0.1 & 0.1 & 0.1 & 0.2 & 0.2 & 0.2 & 0.2 & 0.2 & 0.2 \\
\hline
\end{tabular}

as narrow-bands centered on the $9.7 \mu \mathrm{m}$ silicate feature and two strong PAH bands accessible from ground at 8.7 and $11.6 \mu \mathrm{m}$.

The high-resolution data confirm the presence of a compact mid-infrared source coinciding with IRS 1. An unresolved point source (size $\lesssim 0.5 \operatorname{arcsec} F W H M$ ) accounts for the bulk $(\sim 80 \%)$ of emission. The image obtained in the $11.6 \mu \mathrm{m}$ PAH band shown in Fig. 10 shows the presence of a faint extended emission region towards the north of the stellar source. Optical broadband photometry of the source in the Johnson $B V R I$ bands have been performed in order to cover the spectral energy distribution of the stellar photosphere of IRS 1. Table 6 presents our optical and mid-infrared photometry of IRS 1.

\section{Discussion}

\subsection{Evidence for a pre-protostellar core in the center of the dark cloud}

Our submillimeter observations towards the central region of the dark cloud complex have revealed the presence of a centrally condensed, dense $\left(n\left(\mathrm{H}_{2}\right)=2 \times 10^{5} \mathrm{~cm}^{-3}\right)$, very cold $\left(T_{\mathrm{d}} \sim 12 \mathrm{~K}\right)$ and compact $(R \sim 0.2 \mathrm{pc})$ core with a decreasing temperature gradient towards the center. Similar conditions have been recently observed in FIR studies of nearby preprotostellar cores (Juvela et al. 2001; Ward-Thompson et al. 2002) and are considered as the initial conditions which pertain in clouds from which stars form. Most of the known prestellar cores are however of low-mass. Is it possible that FIR 1 contains a rare example of a massive pre-protostellar core? Even considering that probably more than $50 \%$ of the mass are dispersed during the early phases of star formation, the total mass 


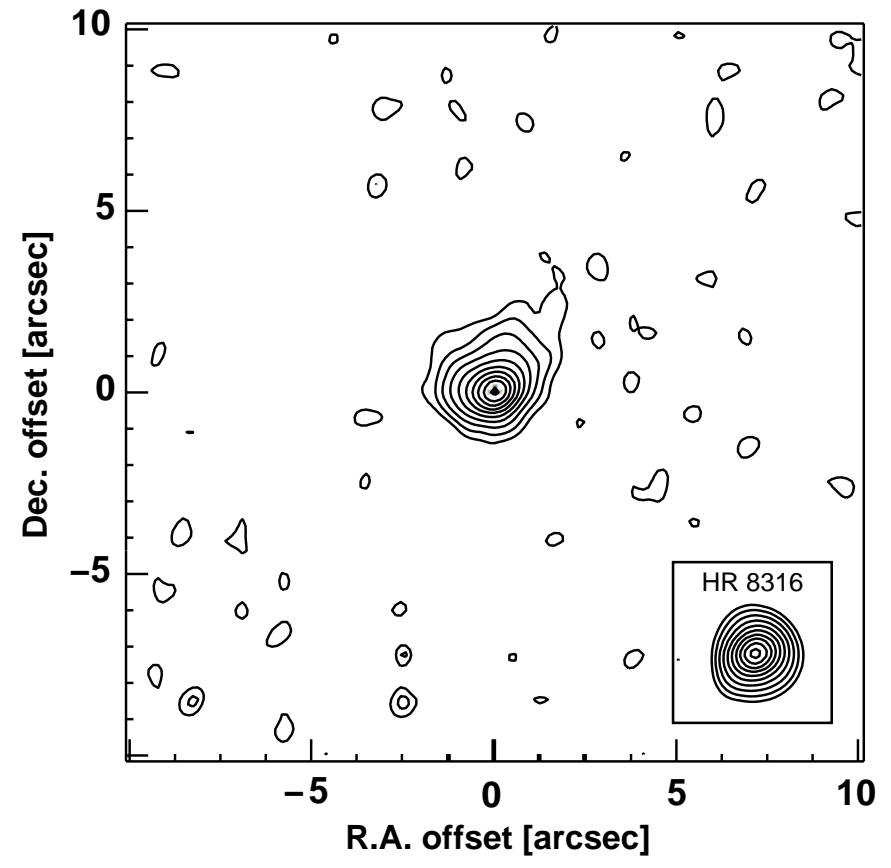

Fig. 10. Diffraction-limited $11.6 \mu \mathrm{m}$ image of the Herbig B2 star IRS 1, obtained with the thermal infrared camera MAX at UKIRT using a narrow PAH-filter. The bulk of the emission originates from an unresolved compact source ( $\operatorname{size} \leq 0.5 \operatorname{arcsec} F W H M$ ). A faint region of extended PAH emission is seen towards the north. An image of the stellar point source HR 8316 is inserted.

of the core $M=50 M_{\odot}$ is likely to be massive enough to form at least one late O- or early B-type star.

By definition a pre-protostellar object has to be gravitationally bound. In order to investigate the dynamical state of the core, we consider the virial theorem. The condition for a gravitationally bound molecular clump or core is given by

$E_{\text {mag }}+E_{\text {pot }}=2 \cdot\left(E_{\text {kin }}-E_{\text {ext }}\right)$,

where $E_{\text {mag }}$ denotes the net magnetic energy, $E_{\text {pot }}$ is the gravitational energy, $E_{\text {kin }}$ the total kinetic energy and $E_{\text {ext }}$ accounts for external pressure. We assume that kinetic energy consists of the thermal energy of the molecules and the energy of their turbulent motion:

$E_{\text {kin }}=E_{\text {therm }}+E_{\text {turb }}=\frac{3}{2} N k T+\frac{3}{2} M \sigma_{\text {turb }}^{2}$.

We derive $\sigma_{\text {turb }}^{2}=\Delta V^{2} /(8 \ln 2)-k T / m$, where $\Delta V$ is the observed line width of our ammonia data and $m$ the mass of the ammonia molecule. The total line width of $\Delta V=0.955 \mathrm{~km} \mathrm{~s}^{-1}$ is an upper limit, since it was derived from a Gaussian fit of the whole maingroup. On the basis on the $(1,1)$ spectrum presented in Fig. 6 it is unclear if the two peak components correspond to the resolved hyperfine structure transition, which would in term lower $\Delta V$, or if the double peak is simply due to noise in the spectrum. The error of the kinetic temperature $T_{\text {kin }}=21.3 \pm 5 \mathrm{~K}$ is rather high, due to the low $S / N$-ratio in the $(2,2)$ spectrum. Usually the kinetic gas temperatures in cold and dense regions are lower than the obtained dust temperatures. We therefore consider $T_{\text {kin }}$ as an upper limit.
Table 7. Physical properties of the cold core in ISOSS J 20298+3559.

\begin{tabular}{lcl}
\hline \hline Mass & $50 \pm 10$ & $M_{\odot}$ \\
Dust Temperature & $12 \pm 2$ & $\mathrm{~K}$ \\
Size & $0.55 \times 0.29$ & $\mathrm{pc}^{2}$ \\
Visual extinction $A_{V}$ & 30 & $\mathrm{mag}$ \\
Column density $N\left(\mathrm{H}_{2}\right)$ & $2.2 \times 10^{22}$ & $\mathrm{~cm}^{-2}$ \\
Density $n\left(\mathrm{H}_{2}\right)$ & $\sim 2 \times 10^{5}$ & $\mathrm{~cm}^{-3}$ \\
Gravitational energy & $6.4 \times 10^{37}$ & $\mathrm{~J}$ \\
\hline
\end{tabular}

With the effective core radius of $R=0.2 \mathrm{pc}$ we derive a gravitational potential of the homogenous ellipsoid (Liljeström 1991)

$E_{\text {pot }}=3 G M^{2} / 5 R$.

We find $E_{\text {pot }}=-6.4 \times 10^{37} \mathrm{~J}, E_{\text {therm }}=1.0 \times 10^{37} \mathrm{~J}$ and $E_{\text {turb }}=2.5 \times 10^{37}$. Relative to $\left|E_{\text {pot }}\right|$ the total kinetic energy sums up to $52 \%$. Neglecting external pressure and magnetic energy for which we have no data at present, we conclude that the cold core is roughly in virial equilibrium. If it will collapse or not, either as a whole or in fragments, will depend mainly on the local turbulent velocity field. Further molecular line data of sufficiently high spatial resolution are needed to assess the collapse issue. Table 7 summarizes the properties of the cold core region.

\subsection{The nature of the two cold submillimeter sources SMM 1 and SMM 3}

Phenomenologically, the youngest protostars (Class 0 objects) are characterized by three attributes (André et al. 1993):

1. Very little emission at $\lambda<10 \mu \mathrm{m}$,

2. Spectral energy distribution similar to a blackbody of $T \sim$ $15-30 \mathrm{~K}$ and

3. $L_{\text {submm }} / L_{\text {bol }}>5 \times 10^{-3}$, where $L_{\text {submm }}$ is the luminosity measured at $\lambda>350 \mu \mathrm{m}$.

We suggest that SMM 1 and SMM 3 fullfil these criteria. SMM 1 and SMM 3 are not detected in the mid-infrared, making them candidates for the youngest protostellar objects in the vicinity of ISOSS J $20298+3559$.

The spectral energy distributions of SMM 1 and SMM 3 are presented in Fig. 11. Relying only on our submillimeter photometry, the spectral energy distributions of both sources can be described by modified blackbodies with dust temperatures between 10 and $38 \mathrm{~K}$. The large uncertainty of the temperature is mainly due to the unknown dust emissivity index $\beta$ which covers the range between $1.0<\beta<2.0$ for protostellar objects and YSOs (Dent et al. 1998). To unambiguously determine the dust emissivity $\beta$, measurements at the maximum or Wien part of the energy distribution are required.

Next we try to provide $60 \mu \mathrm{m}$ upper limits for SMM 1 and SMM 3, considering the flux of FIR 1 minus that of main contributors. Due to confusion with large amounts of distributed cold dust detected by the large-beam IRAS $100 \mu \mathrm{m}$ and ISOPHOT $170 \mu \mathrm{m}$ bands, the photometry at these wavelengths can hardly be used for a reliable characterization of the 

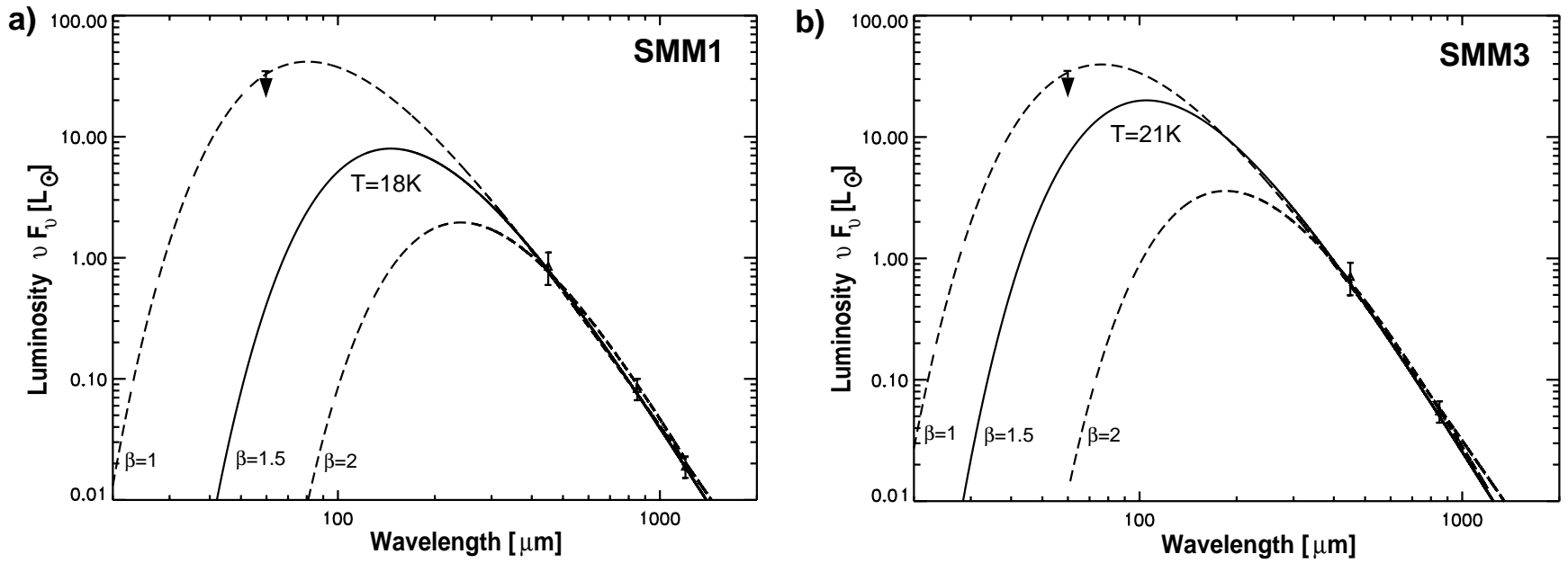

Fig. 11. Spectral energy distribution of the compact submillimeter sources SMM 1 and SMM 3. The temperature is only indicated for $\beta=1.5$. See text for a discussion of the value of the $\beta$-parameters. The arrow indicates a $60 \mu \mathrm{m}$ upper limit based on IRAS photometry.

two faint compact sources. The most stringent upper limit for SMM 1 and SMM 3 can be derived from the IRAS $60 \mu \mathrm{m}$ band. The photometry at this wavelength is however affected by the emission of very small dust particles, transiently heated in the reflection nebula of the Herbig B2 star IRS 1. The presence of such extended PAH and VSG emission is indicated on our midinfrared images of IRS 1 . The IRAS measurements at $12 \mu \mathrm{m}$, $25 \mu \mathrm{m}$ and $60 \mu \mathrm{m}$ are particularly sensitive to such an extended emission component. In order to determine the contribution of thermal radiation of transiently heated PAH and very small grains in these bands we have used the three-phase dust models by Désert et al. (1990). After subtraction of compact emission from IRS1 according to our model (see next section), we determined the relative contributions of PAH and VSG emission from the residual IRAS $12 \mu \mathrm{m}$ and $25 \mu \mathrm{m}$ photometry and using the model by Désert et al. (1990) for the radiation field of a stellar source with $T_{\mathrm{eff}}=20000 \mathrm{~K}$. We find a contribution of $F_{60 \mu \mathrm{m}}=7 \pm 3$ Jy from the Herbig star IRS 1 and its reflection to the total flux density of $F_{60} \mu \mathrm{m}=14 \mathrm{Jy}$. The residual flux density of $F_{60 \mu \mathrm{m}}=7 \mathrm{Jy}$ is considered as an upper limit for each of the two sources.

Using the possible range $1.0<\beta<2.0$ for the emissivity index, we derive total masses of $3.7 M_{\odot}<M<18 M_{\odot}$ for SMM 1 and $2 M_{\odot}<M<8 M_{\odot}$ for SMM 3. The corresponding bolometric luminosities cover the range $36 L_{\odot}>L_{\text {bol }}>1.8 L_{\odot}$ for SMM 1 and $34 L_{\odot}>L_{\text {bol }}>3.3 L_{\odot}$ for SMM 3 respectively. If we favor an intermediate emissivity index of $\beta \sim 1.5$, which was the average value found in the large submillimeter survey of 73 young stellar objects by Dent et al. (1998), then we obtain for the two sources the following masses and luminosities:

SMM 1: $M=8.0 M_{\odot}, L_{\mathrm{bol}}=7 L_{\odot}, T_{\mathrm{d}}=18 \mathrm{~K}$

SMM 3: $\mathrm{M}=3.3 M_{\odot}, L_{\mathrm{bol}}=17 L_{\odot}, T_{\mathrm{d}}=21 \mathrm{~K}$.

We find $L_{\text {submm }} / L_{\mathrm{bol}} \gtrsim 1 \%$ for each choice of $1.0<\beta<$ 2.0, making SMM 1 and SMM 3 bona-fide candidates for Class 0 objects. Further evidence for SMM 1 and SMM 3 being genuine protostars could be provided via detections of a molecular outflow and/or a cm-wavelength continuum source.

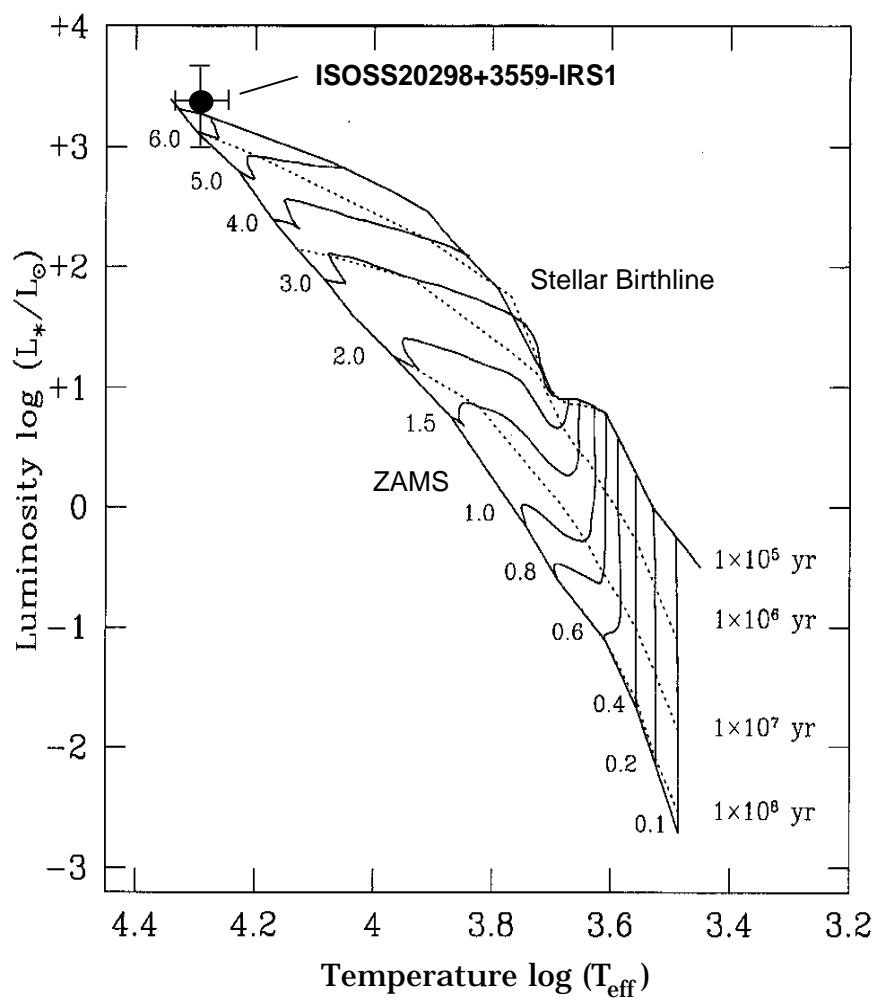

Fig. 12. H-R diagram with stellar effective temperatures and luminosities, adopted from Palla \& Stahler (1999). Stellar masses along the zero-age main sequence (ZAMS) are indicated in units of $M_{\odot}$. The Herbig B2 star IRS 1 is located close to the stellar birthline, which was calculated for an accretion rate of $\dot{M}=10^{-5} M_{\odot} \mathrm{yr}^{-1}$. The pre-mainsequence tracks overlaid indicate a stellar age of less than $40000 \mathrm{yr}$.

\subsection{Evidence for accretion onto the extremely young Herbig B2 star IRS 1}

The Herbig B2e star IRS 1 is the most luminous young stellar object embedded in the cold cloud complex. A very early evolutionary stage of IRS 1 is indicated from its locus in the Hertzsprung-Russel diagram presented in Fig. 12. According to 


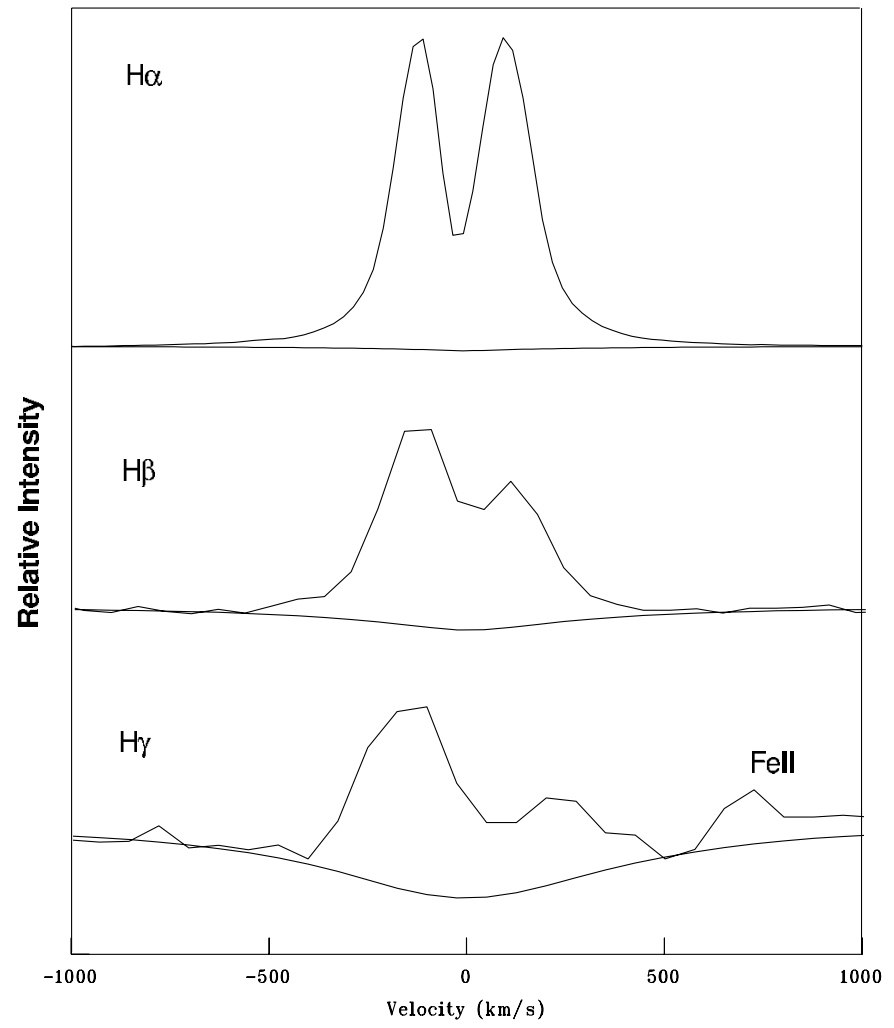

Fig. 13. Emission line profiles of the $\mathrm{H}$ I Balmer series. $\mathrm{H} \alpha, \mathrm{H} \beta$ and $\mathrm{H} \gamma$ are shown from top to bottom. The stellar continuum for a standard star of identical spectral type is shown for each transition. In addition to a strong central self absorption, the redshifted emission line component is suppressed for the higher order lines, indicating infall in an accretion process.

the pre-main-sequence evolutionary tracks by Palla \& Stahler (1993) the source is located close to the stellar birthline computed for $\dot{M}=10^{-5} M_{\odot} \mathrm{yr}^{-1}$ corresponding to a $M_{*}=6.5 M_{\odot}$ star with an age of less than $40000 \mathrm{yr}$ immediately after becoming optically visible.

The observation of inverse $\mathrm{P}$ Cygni profiles as shown by assymetric red and blue line components of the $\mathrm{H}$ I Balmer transitions presented in Fig. 13 provides evidence for ongoing mass infall in a magnetospheric accretion process (Edwards et al. 1994). From the redshifted absorption component in the Si II lines at 6347.1 $\AA$ and $6371.4 \AA$ transitions shown in Fig. 9 we derive an infall speed of $110 \pm 10 \mathrm{~km} \mathrm{~s}^{-1}$.

Near-infrared photometry and our sub-arcsecond resolution mid-infrared images revealed the presence of strongly condensed ( $r \lesssim 250 \mathrm{AU}$ ) warm dust in the immediate surrounding of IRS 1 . In order to model the optical and infrared spectral energy distribution of the heavily reddened source IRS 1, a reliable determination of the extinction towards the source is essential. Based on the effective stellar temperature derived from our observations of photospheric He I lines, we apply a Kurucz model atmosphere with $T_{\text {eff }}=20000 \mathrm{~K}$ for the stellar photosphere. We assume solar metallicity. Our dereddened $B V R I$ photometry is best described for $A_{V}=6.1 \pm 0.3 \mathrm{mag}$ where the error is due to the uncertainty of $T_{\text {eff }}$. Assuming the distance $d=1.8 \mathrm{kpc}$ of the cloud complex we derive a

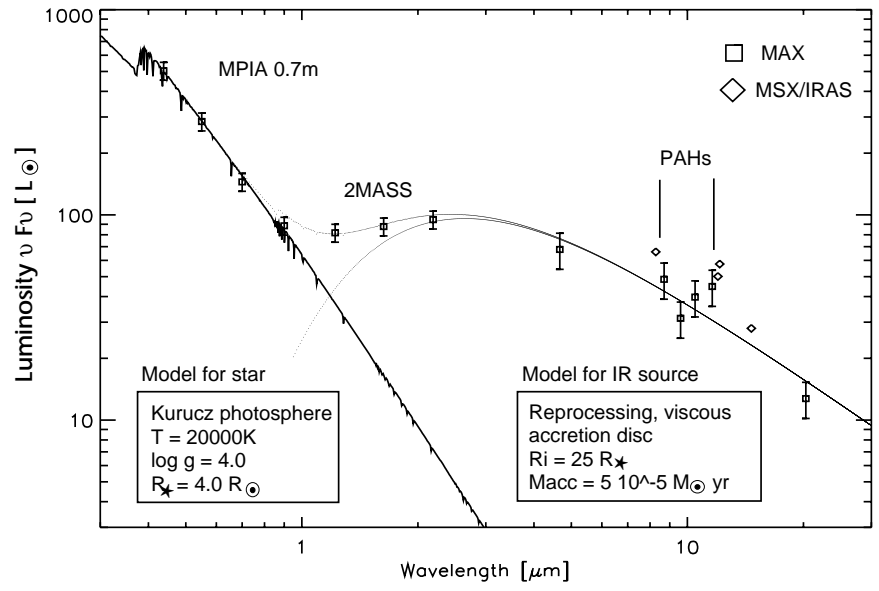

Fig. 14. Dereddened optical and infrared spectral energy distribution of the Herbig star IRS 1. We have modelled the strong infrared excess emission by an optically thick accretion disk. The star is described by a Kurucz model with a $T_{\text {eff }}=20000 \mathrm{~K}$ photosphere $(\log g=4.0)$. Our observations obtained with the MPIA $0.7 \mathrm{~m}$ telescope in the optical and with MAX at UKIRT in the thermal infrared are in good agreement with the model.

stellar radius of $R_{\star}=4 \pm 0.5 R_{\odot}$ from the surface flux density of the Kurucz model. The integrated bolometric luminosity of the photosphere is $L_{\star}=2200 \pm 600 L_{\odot}$.

How can our observations of ongoing accretion and circumstellar dust for this extremely young stellar object be combined in a consistent model? Figure 14 shows the dereddened spectral energy distribution of IRS 1 . The near-infrared emission bump seen at about $3 \mu \mathrm{m}$ wavelength is characteristic for Herbig AeBe stars and has been interpreted either by the presence of a circumstellar disk with an inner hole (Hillenbrand et al. 1992), by spherical envelopes (Hartmann et al. 1993) or the combination of both (Miroshnichenko et al. 1999). While there is general consensus that the Herbig Ae stars are surrounded by circumstellar disks similar to those of T Tauri stars during most of their pre-main-sequence phase (Natta et al. 2001), only one out of seven Herbig Be stars was detected by mm-interferometry (Natta et al. 2000). Extended halos and envelopes in the near- (Leinert et al. 2001) and mid-infrared (Prusti et al. 1994) are more frequently observed around more massive Herbig Be stars. However, these observational differences could be due to a faster evolution of massive stars and differences in the relative timescales of the PMS phases, which limit the detection of disks around early B stars to the very early stage of their evolution.

We suggest that our spectroscopic signatures of ongoing accretion and the infrared excess can best be combined in terms of an accretion disk around IRS 1. We have calculated the spectral energy distribution of an active viscous accretion disk including radiative heating by photospheric radiation from IRS 1 . The radial temperature dependence of the optically thick, geometrically thin disk is then assumed to be $T(r)=\left[T_{\text {acc }}(r)^{4}+\right.$ $\left.T_{\text {rad }}(r)^{4}\right]^{1 / 4}$. We use a standard $\alpha$-disk (Lynden-Bell \& Pringle 1974). Its accretion disk temperature profile is given by

$T_{\mathrm{acc}}(r)=\left[\frac{3 G M_{\star} \dot{M}}{8 \pi \sigma r^{3}}\left(1-\sqrt{\frac{R_{\star}}{r}}\right)\right]^{1 / 4}$. 
Table 8. Properties of the Herbig B2 star IRS 1 and its accretion disk.

\begin{tabular}{lcl}
\hline \hline Mass & $6.5 \pm 1$ & $M_{\odot}$ \\
Luminosity & $2200 \pm 600$ & $L_{\odot}$ \\
Effective temperature & $20000 \pm 2500$ & $\mathrm{~K}$ \\
Radius & $4.0 \pm 0.5$ & $R_{\odot}$ \\
Visual extinction $A_{V}$ & $6.1 \pm 0.3$ & $\mathrm{mag}$ \\
Age & $<40000$ & $\mathrm{yr}$ \\
Rotational velocity & $158 \pm 25$ & $\mathrm{~km} \mathrm{~s}^{-1}$ \\
Inner disk radius & 60.0 & $R_{\odot}$ \\
Accretion rate & $5 \times 10^{-5}$ & $M_{\odot} \mathrm{yr}^{-1}$ \\
\hline
\end{tabular}

In the flat disk approximation its temperature profile due to irradiation only is

$T_{\mathrm{rad}}(r)=\left[\frac{2 T_{\mathrm{eff}}^{4} R_{\star}^{3}}{3 \pi r^{3}}\right]^{1 / 4}$.

Emerging spectral energy distributions have been computed for varying inner disk radii $R_{\mathrm{i}}$ and accretion rates $\dot{M}_{\text {acc }}$. As shown in Fig. 14 the fit of the star+disk SED to the dereddened photometry of IRS 1 is consistent with a dusty, optically thick accretion disk. Table 8 summarizes the model parameters of the system.

Next we consider whether our data could also allow an envelope: our spectrophotometry of IRS 1 obtained in the narrow-band silicate filter at $9.7 \mu \mathrm{m}$ shows a weak absorption feature $\left(\tau_{9.7 \mu \mathrm{m}} \sim 0.2 \pm 0.1\right)$ which is consistent with the total line-of-sight extinction towards the source according to Whittet el al. (1987). Models of spherical envelopes which account for the observed spectral energy distributions of Herbig Be stars all predict strong silicate emission (Berilli et al. 1992; Miroshnichenko et al. 1997). In order to explain the suppression of the silicate emission feature in a B0 star, the inner radius of the envelope must be about 0.02 pc (Natta et al. 2000). This is inconsistent with our diffraction-limited mid-infrared images, which show that the bulk of flux from IRS 1 arises from a true point source, i.e. the radius of the mid-infrared emitting region is $<400 \mathrm{AU}$.

\subsection{Star formation efficiency}

We estimate the star formation efficiency $S F E=M_{\text {stars }} / M_{\text {tot }}$ in the central region ISOSS J 20298+3559-FIR1. $M_{\text {tot }}$ includes the masses of the diffuse component, the dense core and all young stellar objects. We have determined the masses for the near-infrared objects IRS 2...5 from the absolute magnitudemass relation by Carpenter et al. (1993).

Concerning the masses of the Class 0 sources SMM 1 and SMM 3 we assume that they loose $50 \%$ of their envelopes during their further evolution (Bachiller et al. 1996). The mass of all young stellar objects is then $M_{\text {stars }}=20 M_{\odot}$. Adding the mass of gas and dust in FIR 1 to the stellar masses we obtain $M_{\mathrm{tot}}=140 M_{\odot}$. This results in a star formation efficiency of about $14 \%$.

To compare: the SFE is about 2-9\% for the Taurus complex (Cohen \& Kuhi 1979) and 1-9\% in Orion A+B
(Carpenter 2000). ISOSS J 20298+3559-FIR 1 clearly has at least the efficiency of these low- and high-mass star forming regions.

\section{Conclusions}

We have identified the young star forming region ISOSS J 20298+3559 by performing a cross-correlation of cold compact far-infrared sources from the ISOPHOT $170 \mu \mathrm{m}$ Serendipity Survey database with the 2MASS, MSX and IRAS surveys. Multi-wavelength follow-up observations of this region yield:

1. The star forming region is associated with a complex of four optical dark clouds $\mathrm{C} 1$...C4 which have a total mass of $760 M_{\odot}$.

2. We derived a distance of $1800 \pm 300 \mathrm{pc}$ based on optical extinction data. This associates the region with the Cygnus X Giant Molecular Cloud in agreement with our molecular line kinematics.

3. The cold ISOSS source FIR 1 corresponds to the dense inner region of the central dark cloud $\mathrm{C} 1$ and contains a total mass of $120 M_{\odot}$ gas and dust with an average temperature of $16 \mathrm{~K}$.

4. We have identified two candidate Class 0 objects SMM 1 and SMM 3. The sources have masses of 8 and $3.5 M_{\odot}$ which makes them precursors of intermediate mass stars.

5. The externally heated cloud core of $\mathrm{C} 1$ has a total mass of $50 M_{\odot}$ and a central dust temperature as low as $11 \mathrm{~K}$. Ammonia in the $\mathrm{NH}_{3}(1,1)$ transition has been detected. The object is gravitationally bound as derived from our ammonia molecular line observations, which makes it a candidate massive pre-protostellar core.

6. The most luminous object in the vicinity is the Herbig B2 star IRS 1 . The object has a mass of $6.5 M_{\odot}$ and a bolometric luminosity of $2200 L_{\odot}$. Inverse P Cygni profiles in the higher HI Balmer series and SiII lines indicate ongoing accretion. The spectral energy is well described by a Kurucz model for the stellar photosphere and a viscous reprocessing accretion disk. The stellar age inferred from pre-mainsequence evolutionary tracks is less than $40000 \mathrm{yr}$.

7. Several embedded near-infrared sources have been identified. One of them (IRS 7) is surrounded by a mid-infrared reflection nebula.

8. The star formation efficiency in the dense and cold region FIR 1 is about $14 \%$.

Acknowledgements. We thank the referee, Debra Shepherd, for useful comments. The ISOPHOT Data Center at MPIA is supported by Deutsches Zentrum für Luft- und Raumfahrt e.V. (DLR) with funds of Bundesministerium für Bildung und Forschung, grant No. 50QI0201. OK thanks the Wernher von Braun-Stiftung zur Förderung der Weltraumwissenschaften e.V. for financial support. This study made use of the SIMBAD database operated at CDS, Strasbourg, France. HIRES images were provided by the Infrared Processing and Analysis Center. This publication makes use of data products from the Two Micron All Sky Survey, which is a joint project of the University of Massachusetts and the Infrared Processing and Analysis Center/California Institute of Technology, funded by 
the National Aeronautics and Space Administration and the National Science Foundation. This research made use of data products from the Midcourse Space Experiment, funded by the Ballistic Missile Defense Organization with additional support from NASA Office of Space Science.

\section{References}

André, P., Ward-Thompson, D., \& Barsony, M. 1993, ApJ, 406, 122 Andrillat, Y., Jaschek, M., \& Jaschek, C. 1988, A\&AS, 72, 129 Auer, L. H., \& Mihalas, D. 1973, ApJSS, 223, 433

Baars, J. W. M., Genzel, R., Pauliny-Toth, I. I. K., et al. 1977, A\&A, 61,99

Bachiller, R. 1996, ARA\&A, 34, 111

Berrilli, F., Cociulo, G., Ingrosso, G., et al. 1992, ApJ, 398, 254

Bessel, M. S., \& Brett, J. M. 1988, PASP, 100, 1134

Beuther, H., Walsh, A., Schilke, P., et al. 2002, A\&A, 390, 289

Bogun, S., Lemke, D., Klaas, U., et al. 1996, A\&A, 315, L71

Bok, B. J. 1956, AJ, 61, 309

Cambrésy, L., Epchstein, N., Copet, E., et al. 1997, A\&A, 324, L5

Cambrésy, L., Beichman, C. A., Jarrett, T. H., et al. 2002, ApJ, 123, 2559

Carpenter, J. M., Snell, R. L., \& Schloerb, F. P. 1993, ApJ, 407, 657

Carpenter, J. M. 2000, AJ, 120, 3139

Cesaroni, R., Felli, M., \& Testi, L. 1997, A\&A, 325, 725

Cohen, M., \& Kuhi, L. V. 1979, ApJSS, 41, 743

Cohen, M., Witteborn, F. C., Carbon, D. F. 1996, AJ, 112, 2274

Cutri, R. M., Skrutski, M. F., van Dyk, S., et al. 2000, Explanatory Supplement to the 2MASS Second Incremental Data Release

Dame, T. M., \& Thaddeus, P. 1985, ApJ, 297, 751

Dent, W. R. F., Matthews, H. E., \& Ward-Thompson, D. 1998, MNRAS, 301, 1049

Désert, F.-X., Boulanger, F., \& Puget, J. L. 1990, A\&A, 237, 215

Dickman, R. L. 1978, AJ, 83, 363

Eagan, M. P., Price, S. D., Soshir, M. M., et al. 1999, Air Force Laboratory Technical report No. AFRL-VS-TR-1999-1522

Edwards, S., Hartigan, P., Ghandour, L., et al. 1994, AJ, 108, 1056

Evans, N. J., Shirley, Y. L., Mueller, K. E., et al. 2002, in Proc. Hot Star Workshop III: The earliest phases of massive star birth, ed. P. A. Crowther, ASP Conf. Ser.

Finkenzeller, U. 1985, A\&A, 151, 340

Gray, D. F. 1976, The observation and analysis of stellar photospheres (Wiley, New York)

Hamann, F., \& Persson, S. E. 1992, ApJSS, 82, 285

Harju, J., Walmsley, C. M., \& Wouterloot, J. G. A. 1993, A\&AS, 98, 51

Hartmann, L., Kenyon, S., \& Calvet, N. 1993, ApJ, 407, 219

Herbig, G. H. 1960, ApJSS, 4, 337

Herbst, W., \& Sawyer, D. L. 1981, ApJ, 243, 935
Hillenbrand, L. A., Strom, S. E., Vrba, F. J., et al. 1992, ApJ, 397, 613

Holland, W. S., Robson, E. I., Gear, W. K., et al. 1999, MNRAS, 303, 659

Horne, K. 1986, PASP, 98, 609

Juvela, M., Mattila, K., Lehtinen, K., et al. 2002, A\&A, 382, 583

Keel, W. 1991, PASP, 103, 723

Kreysa, E., Gemuend, H.-P., Gromke, J., et al. 1998, Proc. SPIE, 3357, 319

Leinert, Ch., Haas, M., Ábrahám, P., et al. 2001, A\&A, 377, 1098

Lemke, D., Klaas, U., \& Abolins, J. 1996, A\&A, 315, L64

Liljeström, T. 1991, A\&A, 244, 483

Lynden-Bell, D., \& Pringle, J. E. 1974, MNRAS, 168, 603

Meyer, M. R., Calvet, R., \& Hillenbrand, L. A. 1997, AJ, 114, 288

Miroshnichenko, A., Ivezic, Z., \& Elitzur, M. 1997, ApJ, 475, L41

Miroshnichenko, A., Ivezic, Z., Vinkovic, D., et al. 1999, ApJ, 520, 115

Molinari, S., Testi, L., Brand, J., et al. 1998, ApJ, 505, L39

Molinari, S., Brand, J., Cesaroni, R., et al. 2000, A\&A, 355, 617

Monet, D. 1996, BAAS, 28, 905

Myers, P. C., \& Benson, P. J. 1983, ApJ, 266, 309

Natta, A., Grinin, V. P., \& Mannings, V. 2000, in Protostars and Planets IV, ed. V. Mannings, A. P. Boss, \& S. S. Russell (Tuscon: Univ. of Arizona Press), 559

Natta, A., Prusti, T., Neri, R., et al. 2001, A\&A, 371, 186

Odenwald, S., \& Schwartz, P. 1993, ApJ, 405, 706

Ossenkopf, V., \& Henning, Th. 1994, A\&A, 291, 943

Palla, F., Brand, J., Comoretto, G., et al. 1991, A\&A, 246, 249

Palla, F., \& Stahler, S. 1993, ApJ, 418, 414

Palla, F., \& Stahler, S. W. 1999, ApJ, 525, 772

Price, S. D., Egan, M. P., Carey, S. J., Mizuno, D. R., \& Kuchar, T. A. 2001, AJ, 121, 2819

Prusti, T., Natta, A., \& Palla, F. 1994, A\&A, 292, 593

Pudritz, R. E. 2002, Science, 295, 68

Rieke, G. H., \& Lebofsky, M. J. 1985, ApJ, 288, 618

Robberto, M., \& Herbst, T. M. 1998, SPIE, 3354, 711

Robin, A., \& Creeze, M. 1986, A\&A, 157, 71

Savage, B. D., \& Mathis, J. S. 1979, ARA\&A, 17, 73

Schlegel, D. J., Finkbeiner, D. P., \& Davies, M. 1998, ApJ, 500, 525

Shepherd, D. S., Yu, K. C., Bally, J., et al. 2000, ApJ, 535, 833

Sodroski, T. J., Odegard, N., Arendt, R. G., et al. 1997, ApJ, 480, 173

Stetson, P. B. 1987, PASP, 99, 191

Stickel, M., Lemke, D., Klaas, U., et al. 2000, A\&A, 359, 865

Thé, P. S., de Winter, D., \& Pérez, M. R. 1994, A\&ASS, 104, 315

Ungerechts, H., Umbanhowar, P., \& Thaddeus, P. 2000, ApJ, 537, 221

Walmsley, C. M., \& Ungerechts, H. 1983, A\&A, 122, 164

Ward-Thompson, D., André, P., \& Kirk, J. M. 2002, MNRAS, 329, 257

Whittet, D., Bode, M. F., \& Murdin, P. 1987, VA, 30, 135

Wood, D. O. S., \& Churchwell, E. 1989, ApJ, 340, 265 